\title{
The importance of sustained compliance with physical distancing during COVID-19 vaccination rollout
}

\author{
Alexandra Teslya ( $\sim$ a.i.teslya@umcutrecht.nl) \\ UMC Utrecht \\ Ganna Rozhnova \\ University Medical Center Utrecht https://orcid.org/0000-0002-6725-7359 \\ Thi Mui Pham \\ Daphne van Wees \\ Hendrik Nunner \\ Noortje Godijk \\ https://orcid.org/0000-0002-6976-6368 \\ Martin Bootsma \\ Utrecht University \\ Mirjam Kretzschmar
}

\section{Article}

Keywords: Covid-19, Vaccine, Mathematical Model, Behavioural Response, Non-pharmaceutical Interventions, Physical Distancing Measures

Posted Date: February 21st, 2022

DOl: https://doi.org/10.21203/rs.3.rs-390037/v3

License: (a) (i) This work is licensed under a Creative Commons Attribution 4.0 International License. Read Full License

Additional Declarations: Yes there is potential Competing Interest. We would like to disclose that one of authors, Dr Ganna Rozhnova, is Editorial Board Member of Communications Medicine.

Version of Record: A version of this preprint was published at Communications Medicine on November 19th, 2022. See the published version at https://doi.org/10.1038/s43856-022-00207-3. 


\title{
The importance of sustained compliance with physical distancing during COVID-19 vaccination rollout
}

\author{
Alexandra Teslya, $\mathrm{PhD}^{* 1}$, Ganna Rozhnova, $\mathrm{PhD}^{\dagger 1,2}$, Thi Mui Pham ${ }^{1}$, Daphne A \\ van Wees, $\mathrm{PhD}^{1}$, Hendrik Nunner ${ }^{3}$, Noortje G Godijk ${ }^{1}$, Martin Bootsma, $\mathrm{PhD}^{1,4}$, \\ and Mirjam E Kretzschmar, $\mathrm{PhD}^{1}$ \\ ${ }^{1}$ Julius Center for Health Sciences and Primary Care, University Medical Center \\ Utrecht, Utrecht University, Utrecht, The Netherlands \\ ${ }^{2}$ BioISI-Biosystems \& Integrative Sciences Institute, Faculdade de Ciências, \\ Universidade de Lisboa, Lisboa, Portugal \\ ${ }^{3}$ Department of Sociology / ICS, Utrecht University, Utrecht, The Netherlands \\ ${ }^{4}$ Faculty of Social Sciences, Utrecht University, Utrecht, The Netherlands
}

January 31, 2022

*Corresponding author:

Dr. Alexandra Teslya

Julius Center for Health Sciences and Primary Care

University Medical Center Utrecht

P.O. Box 85500 Utrecht

The Netherlands

Email: a.i.teslya@umcutrecht.nl

Phone: +31 683890206

†Corresponding author:

Dr. Ganna Rozhnova

Julius Center for Health Sciences and Primary Care

University Medical Center Utrecht

P.O. Box 85500 Utrecht

The Netherlands

Email: G.Rozhnova@umcutrecht.nl

Phone: +31 887553001 


\begin{abstract}
Background: The increasing vaccination coverage against SARS-CoV-2 enabled relaxation of lockdowns in many countries. The public health authorities are seeking recommendations on the continuation of physical distancing measures during ongoing vaccination rollouts. Compliance with these measures is waning while more transmissible virus variants have emerged.
\end{abstract}

Methods: We used a SARS-CoV-2 transmission model to investigate the feedback between compliance, infection incidence, and vaccination coverage. We quantified our findings in terms of cumulative numbers of new infections three and six months after the start of vaccination.

Results: Our results suggest that the combination of fast waning compliance in non-vaccinated individuals, low compliance in vaccinated individuals, low vaccine efficacy against infection and more transmissible virus variants may result in a higher cumulative number of new infections than in a situation without vaccination. These adverse effects can be alleviated by deploying behavioural interventions that should preferably target both vaccinated and non-vaccinated individuals. The choice of the most appropriate intervention depends on vaccination rate and vaccine efficacy again infection.

Conclusion: Supplementary behavioural interventions aiming to boost compliance to physical distancing measures can improve the outcome of SARS-CoV-2 vaccination programmes, until vaccination coverage is suffciently high. For optimal results, these interventions should be selected based on the vaccine efficacy against infection and expected vaccination rate.

Keywords Covid-19 | Vaccine | Mathematical Model | Behavioural Response | Non-pharmaceutical Interventions | Physical Distancing Measures

\title{
Introduction
}

In November 2020, more than one year after the outbreak of COVID-19 was declared a pandemic by the World Health Organisation [1], the state of the pandemic in many countries around the world remained dire, with hospitalisations and death tolls mounting. Amid the second wave that started in fall $2020[2,3,4]$, more transmissible $[5,6,7]$ SARS-CoV-2 variants emerged (e.g., Alpha (B.1.1.7), Beta (B.1.351), and P.1 (Gamma) [8, 9], causing many countries to reinforce physical distancing measures in order to maintain healthcare capacities and to prevent deaths caused by COVID-19. Since then, an even more infectious virus variants, Delta (B.1.617.2) and Omicron (B.1.1.529), emerged. Delta became dominant in Europe [10] and the US [11] in the summer 2021, and caused new pandemic waves. As of winter 2022, Omicron is on its way to replace Delta as the dominant SARS-CoV-2 virus variant in Europe [12] and in the US [11]. These events underscore that the physical distancing measures, while effective in significantly reducing SARS-CoV-2 transmission during the first wave $[13,14,15,16,17]$, alone are not sufficient to limit SARS-CoV-2 transmission and to eradicate the need for future lockdowns, and further measures such as rigorous vaccination campaigns are required.

Fortunately, on the eve of spread of the Alpha variant in Europe, COVID-19 vaccines developed by BioNTech/Pfizer, Moderna, Johnson \& Johnson (Janssen), and AstraZeneca were approved by EMA [18]. FDA approved the first three vaccines for use in the US [19]. Thus, hopes for the end of lockdown periods and relaxation of physical distancing measures were fuelled. Phase 3 randomised clinical trials reported promising vaccine efficacies for preventing laboratory-confirmed 
symptomatic SARS-CoV-2 infection of $(62-92 \%)$ [20, 21, 22]. On 29 March 2021 CDC released a report that the Pfizer/BioNTech and Moderna vaccines have at least $80 \%$ effectiveness in preventing COVID-19. These findings are consistent with earlier reports that the three vaccines (Pfizer/BioNTech, Moderna and AstraZeneca) have some effectiveness in blocking SARS-CoV-2 transmission [23, 24, 25].

Following the start of the vaccination rollout in countries around the world, data was collected which showed that the approved vaccines have high efficacy in conferring immunity against infection acquisition (80-95\% for Pfizer/BioNTech and Moderna [26, 27, 28], and 76\% for Johnson \& Johnson [29]). These results were estimated from data collected between December 2020 and April 2021 in the USA. During this period, the original variant and the Alpha variant (B.1.1.7) were the dominant circulating variants.

Since then, a new, more infectious variant, Delta (B.1.617.2), has emerged and became dominant in many European countries [10] and the USA [30]. A recent study based on data from Israel estimated a significant reduction of BNT162b2 efficacy for the Delta variant in preventing infection, which was $64 \%$ after two doses [31]. This estimate was supported by another report based on the data in a highly vaccinated health system workforce of California San Diego Health [32]. Thus, the understanding of how the deployment of vaccines can impact transmission of SARS-CoV-2 is complicated by the emergence of the new variants $[33,34,35]$. To slow down the appearance rate of antigenically relevant mutations that may escape protection conferred by existing vaccines as well as to reduce the death toll and the burden on healthcare system, a swift and rigorous vaccination campaign seems of utmost importance.

Vaccination rollout, however, faces multiple challenges. Public health services may be confronted with structural and logistical obstacles (e.g., sufficient supply size, capacity to administer shots $[36,37,38]$ ) illustrated by diverging vaccination rates among different countries [39]. Another factor that may affect vaccination rollout is vaccine acceptance [37] that varies greatly across countries from $23.6 \%$ in Kuwait to $97 \%$ in Ecuador [40, 41].

On the other hand, mass vaccination may also have undesirable consequences such as reducing compliance with physical distancing measures. For example, there is evidence that in the Netherlands, in the year following the vaccination rollout, the compliance in the general population, and in the vaccinated population specifically, decreased as compared to the epidemic period where vaccines were not available [42]. For vaccinated individuals, this change in the behavior may happen since following the vaccination event, they perceive COVID-19 to pose a lower risk for them. Moreover, while some individuals get vaccinated to reduce their risks of disease and to limit the transmission in the community, others may do this to gain admittance to public venues. For example, in the Netherlands, many individuals were vaccinated that did not perceive the threat of SARS-CoV-2 infection as high, and instead did it for other reasons, for example, to obtain a QR code (CTB) needed to access public spaces such as restaurants and entertainment venues [43]. On the other hand, the non-vaccinated individuals may also become less compliant with the physical distancing measures, relying on decreased transmission due to the growing vaccination coverage. This is corroborated by the health belief model [44] which posits that adoption of self-protective measures is motivated by perceived susceptibility to becoming infected, among other factors. This perceived susceptibility changes dynamically with evolving epidemiological situation, as evidenced by data collected in the Netherlands [45]. Hence, compliance may increase as the epidemic grows and decline with increasing vaccination coverage. A number of modeling studies have shown that the feedback between the epidemic dynamics and human behaviour has an important role in the disease transmission [46, 47, 48]. In an earlier modeling study [48], we showed that relaxation of compliance with physical distancing measures beyond a threshold may cause a significant in- 
crease in new infections and hospitalisations. This concern is especially relevant at the start of the vaccination campaign, when vaccination coverage is still low.

We developed a socio-epidemiological model (Figure 2) for SARS-CoV-2 transmission to investigate the effects of waning of compliance with physical distancing measures on the dynamics of SARS-CoV-2 transmission as vaccine is rolled out in the population. The transmission dynamics is modelled through a susceptible-exposed-infectious-recovered (SEIR) framework. The vaccine works as all-or-nothing conferring perfect protection against infection acquisition to a fraction of susceptible individuals who receive it. The vaccine delivered to individuals in other disease stages has no effect.

We assume that the vaccination rollout takes place during a government-imposed lockdown, whereupon many public venues are closed or operate at a reduced capacity, thus limiting the average number of contacts. Additionally, the government may issue a set of recommendations with respect to physical distancing. Compliance with these recommendations is captured by a reduction in the daily number of contacts relative to the pre-pandemic level of contacts. The nonvaccinated population is divided into individuals who can be more compliant (henceforth referred to as "compliant") and less compliant ("non-compliant") to measures. The reduction in contacts is larger for compliant and smaller for non-compliant populations. On the other hand, we assume that vaccinated individuals perceive themselves protected from COVID-19 and therefore, are no longer compelled to comply with physical distancing measures. Thus they are not affected by the compliance acquisition-loss process and increase their contact rate above that of non-compliant individuals, thereby returning to nearly pre-pandemic level of contacts. Non-vaccinated individuals can move between compliant and non-compliant modes, and the rates of moving depend on the state of the epidemic and on vaccination coverage. Specifically, more individuals become compliant with physical distancing measures as the incidence of SARS-CoV-2 infection cases increases and lose compliance faster as the proportion of vaccinated individuals grows (see Methods).

We considered a baseline scenario without vaccination and several vaccination scenarios. To observe the full spectrum of possible scenarios, we sampled vaccination rate on a wide range, which was based on the observations during the first six months of the vaccination rollout in European countries and Israel [39]. Further, we considered scenarios for three types of SARS$\mathrm{CoV}-2$ variants. The first variant has the transmission potential of the original variant that was circulating in Europe prior to fall 2020. The second variant is a more transmissible, Alpha-like variant (B.1.1.7), that spread in many European countries during the winter of 2020/2021 and became dominant in the spring of 2021 [49]. Finally, we also considered the dynamics of a "hypercontagious" Delta-like variant (B.1.617.2), which, as of August 2021, became the dominant strain in Europe [10]. We investigated the impact of compliance with physical distancing measures on the numbers of infected individuals over the course of the vaccination rollout. We also compared the cumulative numbers of new infections after three and six months into the vaccination programme to the numbers without vaccination. We tested the robustness of our findings to the values we chose for the initial conditions and parameters by performing multivariate sensitivity analyses (see Figure 5 and Supplementary materials). The values for initial conditions and parameters were sampled continuously.

Next, we considered the potential effects of two interventions aimed at improving compliance. The first intervention is targeted at people who have not been vaccinated yet and aims at keeping their compliance with physical distancing at the level of prior to vaccination rollout. The second intervention is targeted at people who have been vaccinated and aims at keeping their contact rates low. We also considered a combined intervention where both interventions are implemented simultaneously. 
Finally, we considered the a scenario where in the case of a sharp rise in prevalence which may occur due to the decline of compliance with physical distancing measures, the government may impose additional physical distancing rules to reduce SARS-CoV-2 transmission. To wit, in the Netherlands, following a sharp increase in the number of detected infections in June of 2021, the government imposed additional measures which aimed to reduce infection transmission and which were in effect for nearly a month (July 10, 2021 to August 13, 2021) [50]. We have investigated outcomes of the combination of the vaccination rollout with a lockdown which initiates when the prevalence of infectious cases surpasses a threshold.

\section{Results}

\section{Compliance and vaccination rollout}

To model the transmission dynamics of SARS-CoV-2 virus, the model was calibrated to the state of the epidemic and the level of compliance with physical distancing measures prior to the start of vaccination in the Netherlands in November 2020. The size of the population that recovered from SARS-CoV-2 infection was set based on seroprevalence data from the serological study in an age-stratified and regionally weighted representative sample of the Dutch population [51, 52]. The estimated seroprevalence was $4 \%$ in June/July of 2020 [51] and increased to 14\% in February 2021 [52]. To account for the effects of the second wave until the start of vaccination (taken in the simulations to be November 2020) we fixed the recovered population at $8 \%$. The proportion of compliant population was set at $65 \%$ using the study on behavioral measures and well-being conducted in the Netherlands by the National Institute for Public Health and the Environment (RIVM) [53]. We have investigated the sensitivity of the outcomes that we collected to the assumed initial conditions.

The vaccination rates were sampled on an interval. The lowest boundary of the interval is based on the data from the first six months of vaccination rollout in Belarus [39], one of the slower vaccinating countries in Europe, and would lead to $10 \%$ of the population to be vaccinated in the first six months of the vaccination rollout. The upper boundary of the vaccination rate interval is based on the vaccination rollout in Israel in the first six months of the vaccination campaign. Sustaining this rate would lead to almost $60 \%$ of the population to be vaccinated six months after the start of the vaccination rollout. To estimate the boundaries of the vaccination rate interval, we applied linear regression to the data from the respective countries at the start of the vaccination rollout [39]. Henceforth, these rates are referred to as "slow" and "fast", respectively. We provide the code as a part of the model suite that we developed to perform the analyses presented in this work. Figure $2 \mathbf{a}$ shows vaccination coverage during the first six months after the start of vaccination rollout for slow and fast vaccination. In our analyses, we considered a wide range of vaccine efficacies with respect to prevention acquisition of the infection, $55 \%$ to $95 \%$. For some of the results in the main analysis we have fixed the vaccine efficacy to $60 \%$ and $91 \%$, however subsequently we explored sensitivity of the outcomes to this parameter in an exhaustive fashion.

We fixed contact rates for compliant and non-compliant individuals such that the effective reproduction number for the original variant prior to vaccination rollout is 1.1, as estimated for the Netherlands in November 2020 [54]. We have assumed that the baseline epidemiological dynamics when each one of the three variants circulate are identical, except for the probability of transmission per contact. Thus, the effective reproduction number for the Alpha-like variant was 1.65, i.e. $50 \%$ higher than for the original variant [6]. We have set the basic reproductive number for the Delta-like 
variant using the estimate of 4.92 [55], which makes it approximately 2 times more transmissible as the original variant. Therefore, the effective reproductive number for the Delta-like variant was approximately equal to 2.2 at the start of the vaccination rollout. The contact rate of vaccinated individuals was assumed to be close to the pre-pandemic rate and 1.5 times higher than the contact rate of non-compliant individuals [56].

In our model, individuals become compliant if there are infectious individuals in the population. The per capita rate of switching to the compliant state is proportional to the incidence of infectious cases (see Methods, Table 1). The rate of moving to the compliant state was fixed in the main analysis. Furthermore, the compliance which has an intrinsic natural decay rate, wanes more rapidly as the vaccination coverage increases. The proportion of compliant population for a constant incidence of infection is shown in Figure $2 \mathbf{b}$ where we used slow and fast vaccination rates from Figure 2a. We used incidence of 16,062 cases per day, based on the number of infectious people in the Netherlands which was approximated by RIVM using hospital admissions and data from the Pienter Corona study [52] in the period used for the model calibration [4]. For slow vaccination, three months after the start of vaccination, approximately $89 \%$ of the population is compliant with physical distancing measures and after six months, $84 \%$ is compliant. For fast vaccination, the compliant population decreases more rapidly, with only approximately $54 \%$ and $32 \%$ of individuals being compliant after three and six months, respectively.

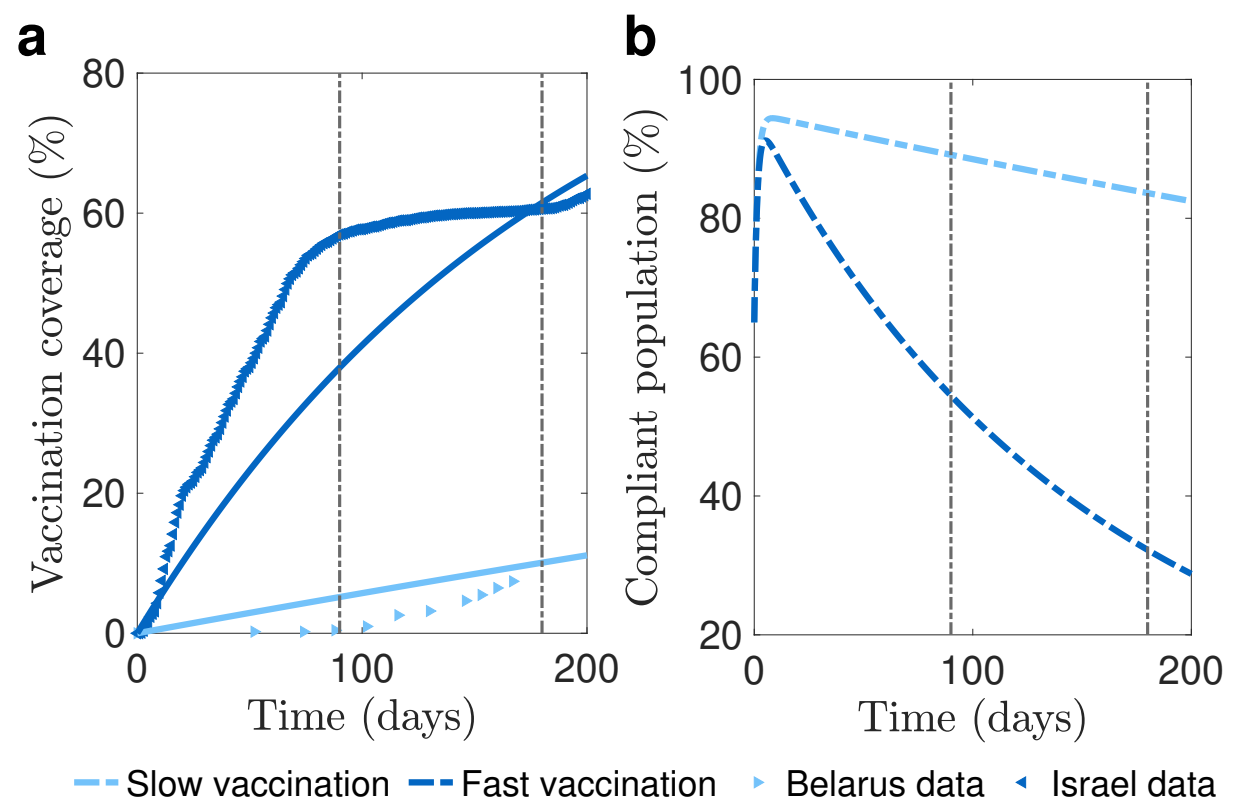

Figure 2: Vaccination coverage and proportion of compliant population during the vaccination rollout. a Increase in vaccination coverage for slow (light blue line) and fast (dark blue line) vaccination rates. Stars show data for Belarus (light blue) and Israel (dark blue) [39], respectively. $\mathbf{b}$ Decrease in the proportion of compliant population for slow and fast vaccination and a fixed incidence of infection (16,062 cases per day) observed in the Netherlands in the period used for the model calibration. Vertical brown lines mark three and six months since the start of vaccination.

The reason for the decline of compliance observed in Figure $2 \mathbf{b}$ is two-fold. First, as the vaccination coverage increases, the compliance in the non-vaccinated population decreases. Moreover, the speed of this decrease depends on how fast vaccination is rolled out. Second, per our assumption, 
vaccinated people perceiving themselves protected from COVID-19, subsequently are no longer compelled to comply with physical distancing. These two processes translate into varying proportions of the compliant population depending on both the incidence of infection and vaccination coverage.

\section{Epidemic dynamics with vaccination}

The model predicts that depending on the speed of the vaccination rollout and transmissibility of the dominant virus variant, as a result of decreasing compliance with physical distancing measures, the prevalence of infected individuals in the presence of vaccination can be temporarily higher than the prevalence in a situation without vaccination (Figure 3). Whether this occurs depends on a number of factors, for example, vaccine efficacy in conferring the immunity against infection acquisition. If a temporary increase in prevalence appears, it is more pronounced for the more transmissible Alpha-like and Delta-like variants than for the original variant (Figures $3 \mathbf{a}, 3 \mathbf{b}$, and 3c). We quantify it as the difference in the cumulative number of new infections relative to the no-vaccination scenario level three and six months after the start of the vaccination rollout.

If the original variant is circulating (Figures $3 \mathbf{a}$ and $3 \mathbf{d}$ ), vaccination can reduce the prevalence below the level of the no-vaccination scenario almost instantaneously (Figure 3a, green curves) or it may take as much as six months after the start of the campaign (Figure 3a, blue curves).

If the vaccine efficacy is high, the effect of the vaccination campaign is expected to be positive with prevalence decreasing below the no-vaccination scenario almost immediately, such that larger reductions in the cumulative number of new infections relative to the no-vaccination scenario are expected for a faster vaccination rate (Figure $3 \mathbf{d}$, green bars).

Given a low vaccine efficacy, we observe a reverse situation for both vaccination rates due to the decline of compliance following the growing vaccination coverage. In this case, in the initial stages of the rollout, the transient prevalence can be higher than in the no-vaccination scenario and this increase is larger for faster vaccination rate (Figure 3a, blue curves). Consequently, slow vaccination, if associated with waning of compliance during vaccine rollout, leads to a smaller excess of cumulative infections than fast vaccination at both three and six months time points (Figure 3d, blue bars). However, on the positive side, we observed in our simulations that if vaccination rate is fast then the prevalence decreases below the level of the no-vaccination scenario six months after the start of vaccination rollout. When vaccination rate is slow, the prevalence eventually decreases below the level of the no-vaccination scenario, but it takes more than 600 days (See Supplementary materials, Section 4, Figure 16a).

If a more transmissible variant is circulating (for example, an Alpha-like or a Delta-like) (Figures $3 \mathbf{b}, 3 \mathbf{c}, 3 \mathbf{e}$, and $3 \mathbf{f}$ ) vaccination rollout can cause an almost immediate decrease in the cumulative number of new infections or a decreased compliance with physical distancing measures can lead to an additional peak in prevalence (Figure 3b). In this latter case, similar to the scenario with the original variant, if vaccine efficacy is low, vaccination can initially lead to an increase of cumulative number of new infections compared the no-vaccination scenario (Figures $3 \mathbf{e}$ and $3 \mathbf{f}$ ). This occurs because waning of compliance coincides with an increased transmissibility of the virus. The period when the prevalence is higher as compared to the no-vaccination scenario lasts even longer than for the original variant (Figures $3 \mathbf{a}, 3 \mathbf{b}$ and $3 \mathbf{c}$ ). 

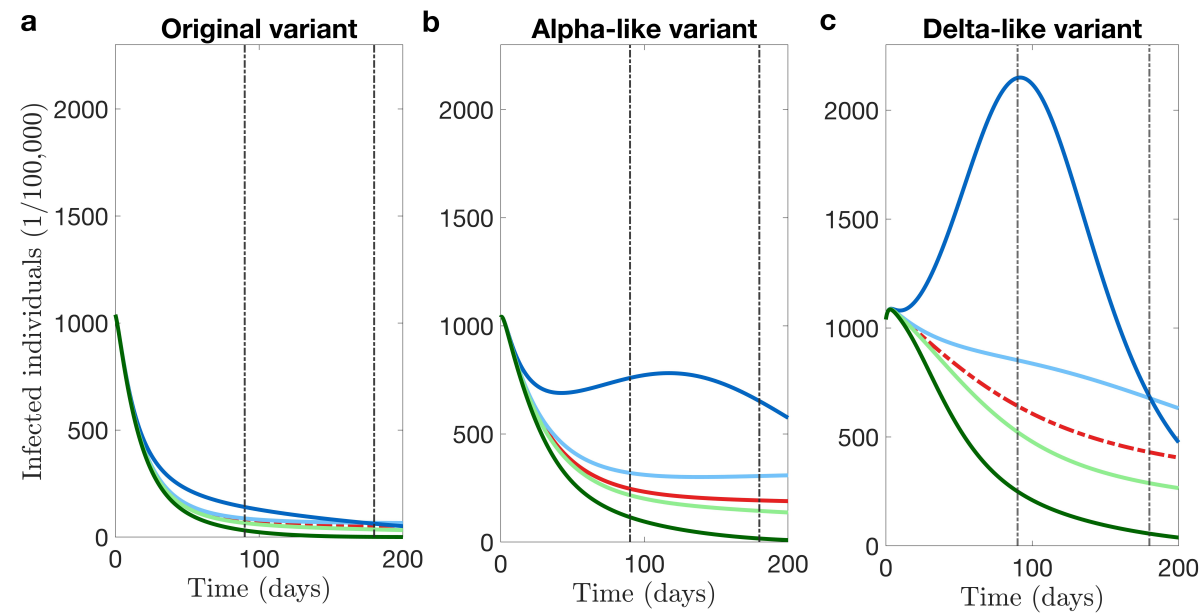

-- Baseline, no vaccination - Fast vaccination, low efficacy

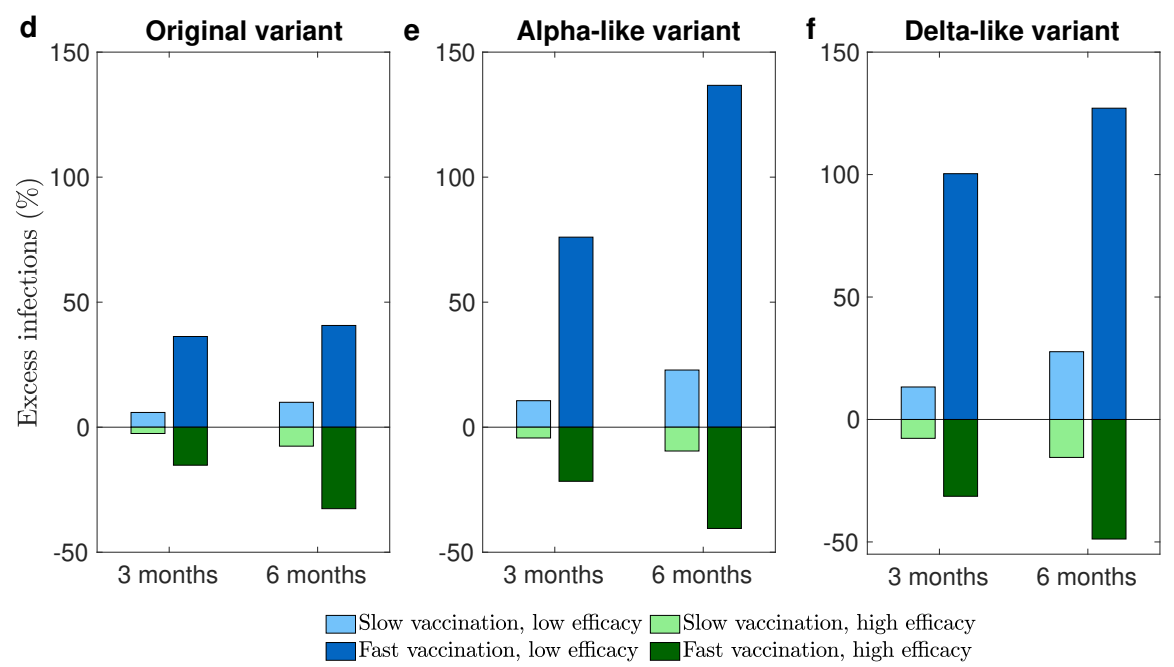

Figure 3: Epidemic dynamics with and without vaccination. a Prevalence of infected individuals versus time when the original variant circulates. b The same output when an Alphalike variant circulates. c The same output when a Delta-like variant circulates. d Difference in the cumulative number of new infections relative to the no-vaccination scenario level for the original variant. e The same output when an Alpha-like variant circulates. $\mathbf{f}$ The same output when a Delta-like variant circulates. $\mathbf{d}$, e and $\mathbf{f}$ show the difference in the cumulative number of new infections relative to the no-vaccination levels when respective variants circulate. Blue curves correspond to a vaccine with $60 \%$ efficacy in conferring protection against infection (low efficacy), green curves correspond to vaccine with $91 \%$ efficacy (high efficacy). In $\mathbf{a}, \mathbf{b}$, and $\mathbf{c}$, vertical brown lines mark three and six months since the start of vaccination.

\section{Contribution of vaccinated and non-vaccinated individuals to the attack rate}

We have seen that for a vaccine with low efficacy a transient increase in prevalence may appear. In what follows we gauge the additional burden this may put on the health system by investigating the role of vaccinated individuals in the transmission dynamics presented in Figure 3 for vaccine with low efficacy. Re-call that the primary goal of COVID-19 vaccine was to reduce the probability of developing a severe disease as a result of becoming infected. Therefore, if a large proportion of infected population has been vaccinated, the temporary rise in prevalence may not necessary 
translate to a significant increase in hospitalization numbers. To identify conditions when this happens, we considered the proportion of infections occurring in the vaccinated population over time (Figure 4) given low efficacy in conferring immunity against infection acquisition (60\%). The analyses show that in the case of slow vaccine uptake (Figures $4 \mathbf{a}, 4 \mathbf{b}$, and $4 \mathbf{c}$ ) vaccinated individuals comprise a small proportion of the infected population even at the end of the six months of the vaccination campaign. Therefore, the increased prevalence among non-vaccinated can be attributed to the decrease of their compliance with physical distancing measures. In the case of fast vaccine uptake, the model predicts that a proportion of infections among vaccinated individuals is higher. Moreover, for a "hyper-contagious" strain, similar to the Delta variant, more than a third of infections are expected to be in the vaccinated population. Thus, the observed rise in the prevalence is in part due to the increased contact rate of susceptible vaccinated individuals. These findings suggest that for slow vaccination the risk of severe disease and death in the population is hardly lowered, while for fast vaccination a significant proportion of the infected individuals will be protected against severe disease, even if the incidence of cases is high. This proportion is higher for a more contagious strain.

\section{Sensitivity of the vaccination rollout outcomes to vaccine efficacy and vaccine uptake rate}

We have seen that depending on factors such as vaccine efficacy, the transient outcomes of vaccination campaign may be very different. Therefore, it is important to investigate systematically the conditions in which a potential negative effects, such as temporary increase in the prevalence above the no-vaccination level, can arise. In this section we present, the findings of the exploration of the joint effects of vaccine efficacy and vaccine uptake rate on the excess of the cumulative number of new infections as compared to the no-vaccination scenario three and six months after the start of vaccination rollout.

Our results are summarized on Figures 5a, 5b and Figures $\mathbf{1 a}, \mathbf{1 b}, \mathbf{2} \mathbf{a}$, and $\mathbf{2 b}$ in Supplementary materials. In all panels, in the region above the magenta curve vaccination rollout yields improvement over the no-vaccination scenario, i.e. the cumulative number of new infections is lower. Importantly, the slower is the vaccination rollout, i.e. the lower is the vaccination coverage after three months of the vaccination rollout, the higher the vaccine efficacy needs to be to avoid an increase of cumulative number of new infections as compared to the no-vaccination scenario. This is a consequence of fast loss of compliance with physical distancing measures as the vaccination coverage grows. Vice-versa, depending on the vaccine efficacy, the speed of the rollout can cause increase or decrease of cumulative number of new infections. If the efficacy is low and the rollout is fast, then initially the cumulative number of new infections is expected to be higher than for the no-vaccination scenario. Moreover, the combination of fast vaccine uptake and low vaccine efficacy is predicted to cause the largest increase in the cumulative number of new infections as compared to the no-vaccination scenario. This happens due to the combined effect of quickly growing vaccination coverage which affects compliance with physical distancing measures in the non-vaccinated population and of increased contact rates of the vaccinated individuals who while potentially protected from the severe disease can still acquire and transmit the infection. However, if the vaccine efficacy is high, given a fast vaccination rate, we expect that the cumulative number of new infections to fall below the level of the no-vaccination scenario. The decrease in the number increases as the vaccination rate increases. We observe that for all variants considered (see

Figures $5 \mathbf{a}, 5 \mathbf{b}$ and Figures $\mathbf{1 a}, \mathbf{1} \mathbf{b}, \mathbf{2 a}$, and $\mathbf{2} \mathbf{b}$ in Supplementary materials), the minimal vaccine efficacy where the cumulative number of new infections decreases over the no-vaccination scenario 
decreases with time since the start of the vaccination rollout.

Slow vaccination
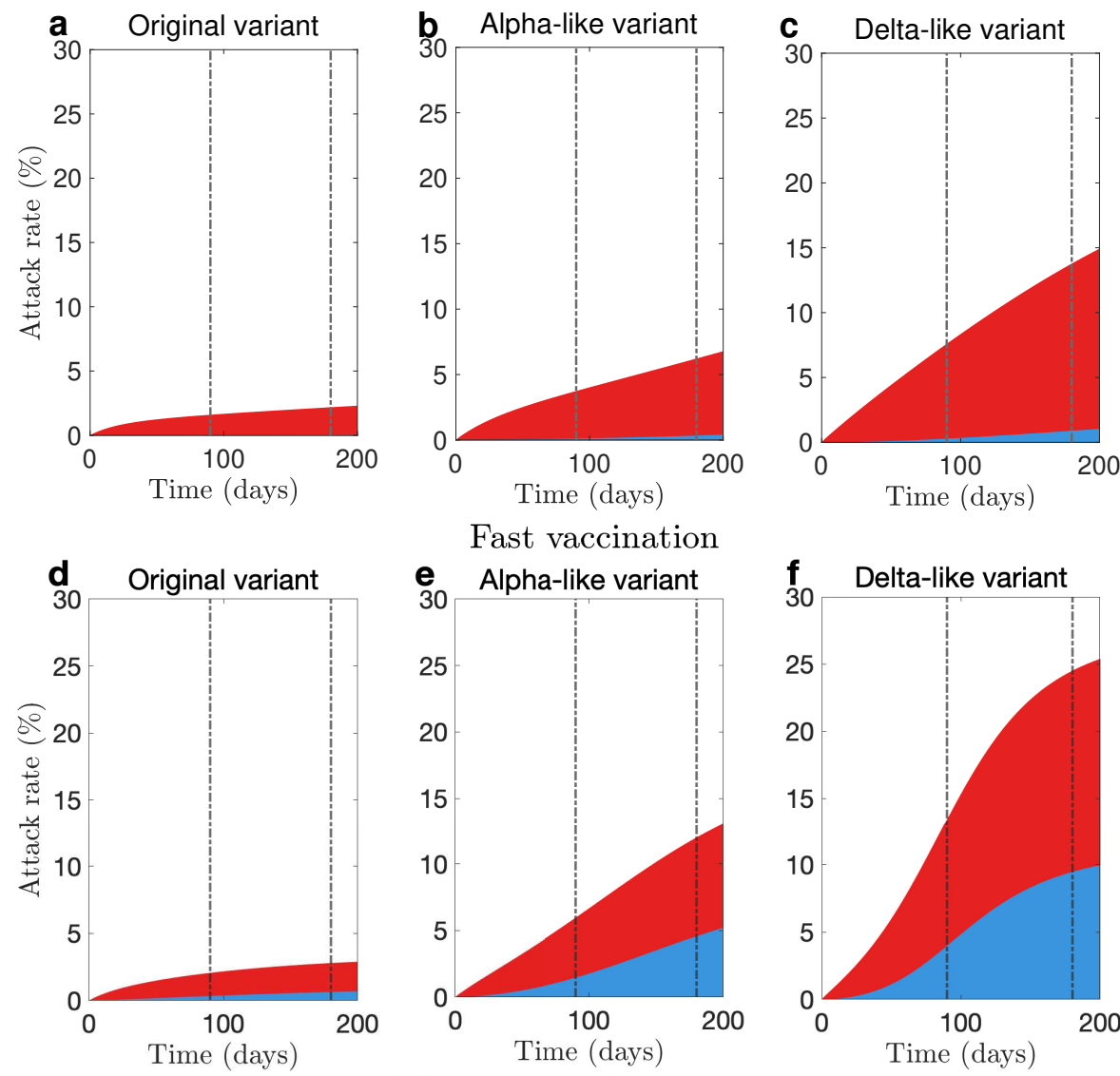

Fast vaccination
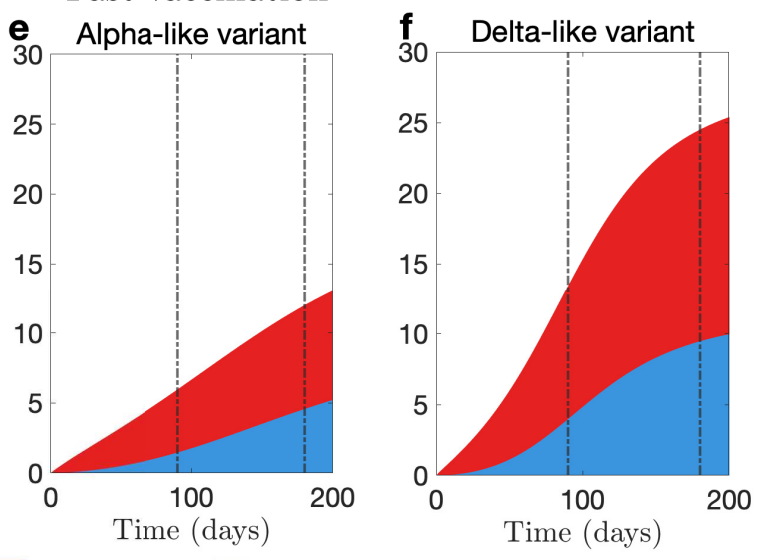

Figure 4: Contribution of vaccinated and non-vaccinated individuals to attack rate during the vaccination rollout given. We consider the scenario with low vaccine efficacy in conferring immunity against acquisition of infection (60\%). a, b, and $\mathbf{c}$ show attack rates versus time given the slow vaccine uptake rate. $\mathbf{d}$, e, and $\mathbf{f}$ show attack rates versus time given the fast vaccine uptake rate. $\mathbf{a}$ and $\mathbf{d}$ show these quantities for the original variant, $\mathbf{b}$ and $\mathbf{e}$ for an Alpha-like variant, $\mathbf{c}$ and $\mathbf{f}$ for a Delta-like variant. Vertical brown lines mark three and six months since the start of the vaccination campaign. Attack rate is the proportion of the population that has been infected until a given time. We adjusted the attack rate so that it describes only new infections that appeared during the time interval that we considered.

The vaccine efficacy where the vaccination campaign does not cause excess infections due to the reduction of compliance is smaller for more transmissible strains, in particular the Delta-like variant (Figures 5a and 5b and Figures $\mathbf{1 a}, \mathbf{1} \mathbf{b}, \mathbf{2} \mathbf{a}$, and $\mathbf{2 b}$ in Supplementary materials).

We refer to the analyses above as to the epidemic dynamics without compliance-targeted interventions. In the following section, we investigated the impact of interventions targeted at maintaining compliance with physical distancing; we compared this to the epidemic dynamics without compliance-targeted interventions and the scenario without either vaccination or interventions. 


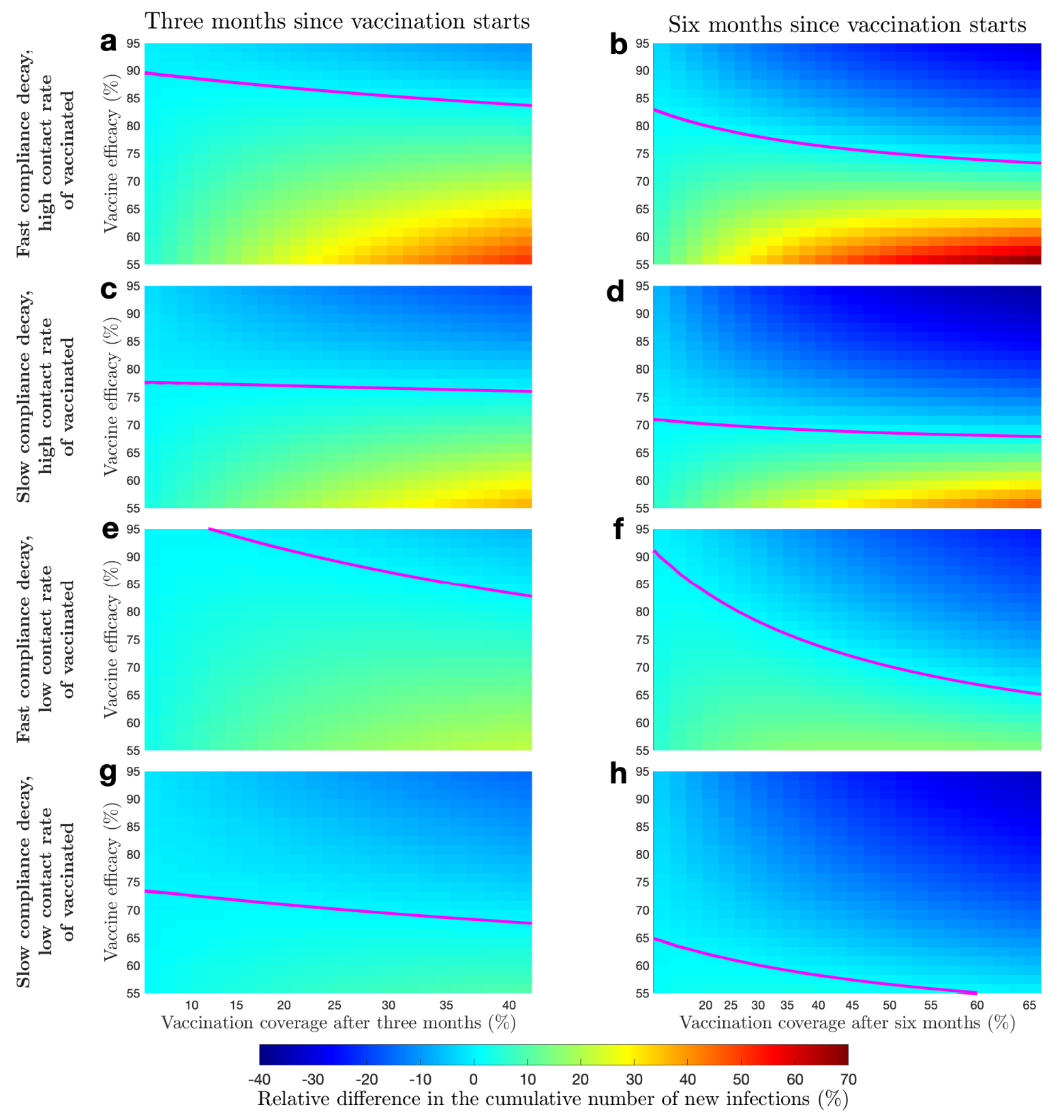

Figure 5: Epidemic dynamics with and without interventions targeting compliance of vaccinated and non-vaccinated individuals. The original variant of the virus circulates. All panels show relative difference in the cumulative number of new infections as compared to the no-vaccination scenario. $\mathbf{a}$ and $\mathbf{b}$ Vaccination rollout not supplemented with compliance interventions three and six months into the vaccination rollout, respectively. $\mathbf{c}$ and $\mathbf{d}$ Vaccination rollout supplemented with compliance interventions targeting non-vaccinated individuals three and six months into the vaccination rollout, respectively. e and $\mathbf{f}$ Vaccination rollout supplemented with compliance interventions targeting vaccinated individuals three and six months into the vaccination rollout, respectively. $\mathbf{g}$ and $\mathbf{h}$ Vaccination rollout supplemented with compliance interventions targeting both vaccinated and non-vaccinated individuals three and six months into the vaccination rollout, respectively. Magenta curves mark boundaries between parameter regions with different sign of the cumulative number of new infections. The scale of $\mathrm{x}$-axis is not linear since the axes were obtained by conversion of the vaccine uptake rate to the vaccination coverage following three and six months after the start of the vaccination rollout. 


\section{Interventions targeting compliance}

To investigate how interventions may improve the impact of vaccination rollout, we considered an intervention that targets compliance of those who are not yet vaccinated and an intervention targeted at the vaccinated population. We assume that the first intervention targets non-vaccinated individuals and is successful in keeping the duration of compliance at the pre-vaccination length (30 days) as vaccination coverage grows. The second intervention, targeted at vaccinated individuals, succeeds in convincing vaccinated individuals to abstain from increasing the contact rate above that of the contact rate of non-compliant individuals. Our model predicts that a successful implementation of either of these interventions reduces the cumulative number of new infections after vaccination rollout and can get this number below the level of the no-vaccination scenario. The effectiveness of these interventions depends on the circulating variant and the vaccine uptake rate. We summarize our findings in Figures 5 and Figures 1 and $\mathbf{2}$ in Supplementary materials.

\section{Intervention 1: targeting compliance of non-vaccinated individuals}

For all three variants, an intervention that targets compliance of non-vaccinated individuals (Figures 5c and 5d and Figures 1c, 1d, 2c, and 2d in Supplementary materials), reduces the minimal efficacy of vaccine required for the cumulative number of new infections after three and six months following the vaccination rollout to be smaller than in the no-vaccination scenario at the respective time points. If there is an excess of new infections (area above the magenta curve), this excess is smaller then the excess number of the vaccination campaign without the compliance-targeting intervention scenario (Figures $5 \mathbf{a}, 5 \mathbf{b}, 5 \mathbf{c}$, and $5 \mathbf{d}$ and Figures 1a-d, 2a-d in Supplementary materials). While for all variants the intervention lowers the vaccine efficacy minimum at which the cumulative number of new infections decreases compared to the no-vaccination scenario, this threshold becomes lower for more transmissible variants. Similarly, the intervention

yields larger relative reductions in the cumulative number of new infections for more transmissible variants.

\section{Intervention 2: targeting compliance of vaccinated individuals}

Effects of this intervention on the cumulative number of new infections depend on the circulating virus variant, vaccine efficacy, and vaccination rate (Figures $5 \mathbf{e}$ and $5 \mathbf{f}$ and Figures $\mathbf{1 e}, \mathbf{1 f}, \mathbf{2 e}$, and 2f in Supplementary materials).

For the original variant and a slow vaccination rate, we observe that after three months of vaccination for the whole range of vaccine efficacies that were considered there is excess of infections as compared to the no-vaccination scenario (Figure 5e). This is contrary to the scenario when the vaccination rollout is not supplemented with compliance-targeted interventions, where vaccinated individuals are characterized by the increased contact rate. This outcome occurs due to the change in mixing. As vaccinated individuals have less contacts, more transmission contacts occur in the non-vaccinated population leading to the increase in the number of infections.

If vaccination rate is fast, three months after the start of the vaccination rollout, we also see mixed results. For the combination of the vaccine efficacy and vaccination rate which gives a decrease in the cumulative number of the new infections in the scenario where vaccination rollout is not supplemented with compliance-targeted intervention, we see this decrease reducing in magnitude. On the other hand, the minimum of vaccine efficacy where the cumulative number of new infections is lower. Finally, the region with excess infections is smaller compared to the scenario where the vaccination rollout is not supplemented with compliance-targeted interventions.

Six months after start of the vaccination rollout, the situation is similar (Figure 5f). Given a slow vaccination rate, the minimum of vaccine efficacy where the relative increase of infections can be avoided is higher than in the scenario where the vaccination rollout is not supplemented with 
the intervention. But if the vaccination rate is fast, than the respective vaccine efficacy minimum is lower than it was without the intervention.

The dynamics for different regions of the vaccine efficacy and vaccination rate for an Alpha-like or a Delta-like variants when the intervention is deployed are qualitatively similar to the dynamics of the original strain (Figures 1e, 1f, 2e, and $\mathbf{2 f}$ in Supplementary materials).

\section{Combination of two interventions}

Finally, combination of the two compliance-targeting interventions leads to improvements that exceed the effects of individual interventions (Figures $5 \mathbf{g}$ and $5 \mathbf{h}$ and Figures $\mathbf{1 g}, \mathbf{1 h}, \mathbf{2 g}$, and $\mathbf{2 h}$ in Supplementary materials). For all three variants, the minimum for vaccine efficacy where the excess of infections as compared to the scenario without compliance-targeted intervention can be avoided, is decreased. Also, excess in the cumulative number of infections is decreased for the region of vaccine uptake rate and vaccine efficacy that we considered. Similar reductions relative to the scenario where the vaccination rollout is not supplemented with compliance-targeting intervention are achieved for the more transmissible variants, provided a fast vaccination rate (Figures $\mathbf{1 g}, \mathbf{1} \mathbf{h}$, $\mathbf{2 g}$, and $\mathbf{2 h}$ in Supplementary materials).

\section{Supplementing vaccination rollout with a lockdown}

Our simulations indicated that due to compliance waning as the vaccination coverage grows, it is possible that an additional prevalence peak appears. So far, in our simulations no centralized intervention triggered by a steep increase in the number of new cases was modeled. Here we consider such an intervention, whereupon if during the vaccination rollout the prevalence of new infectious cases exceeds a certain threshold, the government tightens the lockdown, further restricting the average contact rate, including vaccinated individuals. Once the prevalence falls bellow the threshold, the lockdown is being relaxed to its prior state. We investigated the effect of the threshold prevalence at which the lockdown is initiated on the cumulative number of new infections three and six months after the start of the vaccination campaign (Figures 3-5 in Supplementary materials). We have vaccine efficacy on the range used for the main analysis.

Our simulations indicate that supplementing the vaccination rollout with lockdown which initiates once the prevalence of infectious cases exceeds a threshold can prevent increase of the cumulative number of new infections after three and six of the vaccination rollout as compared to no-vaccination scenario (Figure $\mathbf{3}$ in Supplementary materials). The cumulative number of new infections after three months of the vaccination rollout are larger for the fast vaccination rollout than for the slow. Interestingly, the cumulative number after three months of the vaccination rollout for either vaccination rate is not sensitive to changes in the lockdown strengthening/relaxation threshold on the range that we consider. On the other hand, the cumulative number of new infections after six months of the vaccination rollout for both slow and fast vaccination rates is increasing as the threshold for the strengthening/relaxation of the lockdown grows. While the relative difference in the cumulative number of new infections is larger for a lower lockdown initiation threshold, the gain is not sufficiently large to warrant a strict lockdown that initiates early. The largest decrease in the cumulative number of new infections relative to the no-vaccination scenario happens when the vaccination rate is fast and the vaccine efficacy is high. Decreasing either one of these parameters causes the relative difference to decrease (Figure 4 in Supplementary materials). On the other hand, the largest decrease in the cumulative number of new infections relative to the vaccination rollout without compliance interventions happens when the vaccination rollout is fast and the vaccine efficacy is low (Figure $\mathbf{5}$ in Supplementary materials).

\section{Sensitivity analysis of the epidemic dynamics with vaccination}

We performed sensitivity analysis to test the robustness of our findings to the variations of the initial conditions and parameter values. More specifically, we were interested whether the possi- 
bility of a relative excess in the cumulative number of new infections is preserved across parameter space and how its value changes. In our analyses, unless stated otherwise we considered vaccine with low efficacy (60\%), and varied one parameter at a time while keeping the initial conditions and parameter values fixed to the values that were used to produce simulations summarized on Figure 3 and Figure 17 in Supplementary materials. We considered the dynamics of the original variant. For the ranges used in Sensitivity analysis see Table 1.

Given vaccine with low efficacy, the possibility of the relative increase in the cumulative number of infections after three and six months of the vaccination rollout as compared to the no-vaccination scenario is preserved across the intervals for the initial conditions that we considered (Figures 6-9 in Supplementary materials). The relative difference in the cumulative number of new infections has the highest sensitivity to the initial state of the seroprevalence as compared to the initial conditions in other compartments.

Both the absolute value of the cumulative number of new infections and the relative difference in the number as compared to the no-vaccination scenario are very sensitive to the contact rates of compliant and non-compliant individuals (Figures 12 and 13 in Supplementary materials). The cumulative number of new infections after three months of the vaccination rollout is more sensitive to both than the cumulative number after six months. The cumulative number of new infections is increasing when either one of the contact rates is growing and is highest in the scenario where the average contact rate of the population was close to the pre-pandemic level. The cumulative number of new infections decreases below no-vaccination scenario when the contact rate of compliant individuals is sufficiently close to the contact rate of non-compliant individuals. This happens since the average contact rate of non-vaccinated individuals does not grow significantly even as the growing vaccination coverage causes a decrease in the proportion of compliant population. From Figure 4 we observe that when the original variant circulates, new infections occur mainly among non-vaccinated individuals. This is different for a more transmissible variant such as Delta, where vaccinated individuals contribute significantly to the cumulative number of new infections.

The cumulative number of new infections and the relative difference in the cumulative number of new infections (as compared to the no-vaccination scenario) are very sensitive to variations of the rate of moving to the compliant state and the duration of the compliant state (Figures 14 and 15 in Supplementary materials). The cumulative number of infections is the highest when individuals move to the compliant state at a slow rate but the duration of the compliant state is low (Figure 14). As the rate of moving to compliant state and the duration of being compliant increase the, cumulative number of infections decreases. The relative difference in the cumulative number of infections has the opposite relationship with the two parameters (Figure 15). Such that, the difference is largest when the rate of moving to compliant state is fast and the average duration of staying of compliant is long. We observe that the duration of compliant state has little effect on the possibility of excess infections as compared to the no-vaccination scenario. However, if the rate of moving to compliant state is sufficiently high, the cumulative number of infections will exceed the no-vaccination scenario level.

For the description of methodology and the complete treatment of this topic see Supplementary Materials.

In summary, we gain the following insights: (a) If vaccine efficacy exceeds a threshold, decrease in prevalence is expected to occur almost immediately; (b) If vaccine efficacy is below that value and vaccinated and non-vaccinated individuals relax their compliance with physical distancing measures, an excess in the cumulative number of new infections may occur; (c) In this case, fast vaccine uptake rate may not be advantageous as, combined with diminished compliance, it may lead to a significant relative increase in the number of infections; (d) For all variants that we 
considered, an intervention targeting the non-vaccinated population is effective in reducing the number of infections below the no compliance-targeted intervention scenario and reduces the minimum value for vaccine efficacy necessary to lower this number below the no-vaccination scenario level. However, this threshold is still high; (e) In contrast, the intervention that targets compliance of vaccinated individuals can yields good results even when vaccine efficacy is low, provided fast vaccination rate. This intervention performs better in a long run than in short run; (f) Slow vaccination with a combined compliance-targeting intervention can reduce numbers of infections as compared to the no-interventions scenario. But in order to reduce the number of infections below the level of the no-vaccination scenario, vaccine efficacy should exceed $65 \%$; fast vaccination with a combined intervention reduces the number of new infections even for lower vaccine efficacy.; (g) Strengthening of the lockdown triggered by the rise in prevalence is another intervention that can prevent increase in the cumulative number of new infections. Our results indicate that the initiation threshold for the lockdown can be sufficiently high, thus potentially allowing for shorter periods of the slowing down of the economy.

\section{Discussion}

Using a compartmental model for the spread of SARS-CoV-2 in a population, where physical distancing measures are in place, we investigated the impact of declining compliance with physical distancing measures as vaccination is rolled out on the numbers of infections. One of the key features of our model is a distinct treatment of the loss of compliance by vaccinated and nonvaccinated populations, each of which can relax the compliance of physical distancing measures to a different degree. Additionally, we extended the compliance process to the whole population and not only the susceptible individuals, which qualitatively affects mixing patterns in the population.

Our main finding is that, if compliance decays as the vaccination coverage grows, the speed of vaccination rollout has a strong impact on whether the cumulative number of new infections can be decreased three and six months after the start of vaccination below the level that would have been expected without vaccination (Figure 3). The outcome will depend on the vaccine efficacy. If vaccine efficacy in conferring protection against infection is low, it may lead to an increase in the prevalence exceeding the prevalence in a situation without vaccination and, in the short term, we may even see an additional epidemic peak. Moreover, in the short term, worse outcomes can be expected for a faster vaccination rate. This effect happens due to the loss of compliance by vaccinated individuals. On the other hand, if vaccine efficacy is relatively high, these detrimental effects can be avoided. Moreover, the decrease in prevalence will be larger for faster vaccination rates. However, we note that the available real world data suggests that even in the case when vaccine has a high efficacy, if the physical distancing measures during the initial period of the rollout are relaxed below a threshold, a new wave of infections may happen [57]. The authors of this study analysed the data on vaccinations and COVID-19 incidence collected in Cyprus and Malta between December 2020 and June 2021 during vaccination rollout and observed that Cyprus which supplemented vaccination rollout with strict public distancing measures saw a decline in new cases, despite its vaccination uptake rate being slower than in Malta. Malta, on the other hand, experienced a sharp uptick of new infections in the first stages of vaccination campaign due to more relaxed public distancing measures. Note that should there be an increase in infections relative to the no-vaccination scenario, since among the excess infections a certain proportion of infected people will have been vaccinated, they will have a low probability of developing severe disease or death. 
Finally, as a result of our comprehensive analysis of the effect of the vaccination rate and vaccine efficacy on the cumulative number of new infections, we derived threshold curves which separate parametric regions where the relative difference in the cumulative number of infections as compared to the no-vaccination scenario changes sign (Figure 5). We observed, that if the vaccine has a high efficacy, then the excess of infections can be avoided for a relatively low vaccination uptake rate. As the vaccine efficacy decreases, the uptake rate increases.

In their recently published work Gozzi et al [58] also considered the impact of the feedback between the epidemic dynamics, the vaccination rollout, and compliance with physical distancing on infection transmission dynamics. The authors investigated the effects of waning compliance due to the growing vaccination coverage provided different vaccination strategies and vaccine efficacies across populations with different age contact matrices. Both ours and Gozzi et al [59] qualitative findings are in agreement and are consistent with the results of the earlier studies that have shown that factors that contribute to drastic increase of contact rates (such as vaccination-related behavioral change or premature reduction/removal of non-pharmaceutical interventions) may reduce the benefits of a vaccination programme [60, 61, 62, 63].

Motivated by the conclusions drawn by these studies, we considered the effect of supplementing the vaccination campaigns with communication strategies promoting maintenance of physical distancing behavior aimed at both vaccinated and non-vaccinated individuals and learned that 1) those interventions can significantly improve the outcome of vaccination campaign; 2) the choice of a specific information intervention should be informed by the epidemic circumstance of the situation (such as the dominant variant and speed of vaccination rollout).

An intervention that succeeds in maintaining the compliance with physical distancing in people not yet vaccinated on the same level as before the start of vaccination ensures significant decrease of the cumulative number of new infections throughout. Moreover, for all three virus variants, supplementing vaccination rollout with this intervention reduces the vaccine efficacy threshold for which the cumulative number of new infections is lower than without the vaccination. This effect is seen in both short and long term, but is more pronounced in the long term. The effect for an intervention that targets vaccinated individuals to prevent them from increasing their contact rates after being vaccinated depends on the transmissibility of the dominant variant. If the original variant circulates, the intervention has a positive impact for a fast rollout of vaccination, but cannot avoid detrimental effects of waning of compliance if the vaccination rollout is slow. On the other hand, if the dominating variant has the same transmissibility as Alpha or Delta, then the intervention can improve the outcome of the vaccination rollout over the no-vaccination scenario even when the vaccination rate is slow. Interestingly, for the original and an Alpha-like variant, given a slow vaccination rate, the minimum vaccine efficacy threshold required to avoid a surplus of infections is higher when the vaccination rollout is supplemented with the intervention than when it is not. If a Delta-like variant circulates, supplementing the vaccination rollout with the intervention reduces the threshold for all vaccination rates that we considered. Only the combined effect of both interventions can consistently reduce the cumulative number of new infections below the level of the no-vaccination scenario regardless of the rollout speed (in the vaccination rate range that we considered).

Finally, we compared the effect of compliance-targeting interventions with a centralised intervention that mimics tightening/relaxation of the lockdown when a prevalence threshold is crossed. We observed that the possibility of an excess of infections is eliminated and yields larger decreases in the cumulative number of new infections over the no-vaccination scenario than the compliance-targeting interventions, both in the short term and in the long term. The outcomes of supplementing the rollout with this intervention are not sensitive to the prevalence threshold. 
However, it may come at a price of disrupted social fabric and slowing down of the economy.

Our results are based on some simplifying assumptions, one of them that physical distancing measures remain in place throughout the time period of analysis (six months). While this would be advantageous for preventing transmission of the virus, it might not be feasible out of societal and economic reasons. Therefore, compliance rates may wane even faster in real populations and contact rates may be up to higher, possibly pre-pandemic values during the rollout of vaccination. We do not expect that this would change our results much, as our results are obtained relative to the no-vaccination scenario, which would similarly be affected by a change in physical distancing measures. We expect therefore that the relative effects of vaccination would remain similar as in our simulations. We also assumed that the speed of vaccination rollout stays constant over the time period of six months, which is not the case in reality. In the Netherlands for example, vaccination rates have increased substantially after a slow start in January 2021 [39]. These rates will depend on many factors, nevertheless large differences will remain between countries. Finally, we have captured the dependence of rates of becoming compliant and non-compliant on the incidence of new infectious cases and vaccination coverage, respectively, using linear functions. As the vaccination in many countries continues and the population response data is collected, a more precise formulation of the response functions can be obtained. However, our results predominantly depend on the assumed monotonicity of these functions.

Furthermore, our model is relatively simple, not taking into account age structure and heterogeneity in contact patterns. Therefore, we do not attempt to make quantitative predictions on the impact of vaccination, but we provide qualitative insight into possible effects of waning compliance with physical distancing in the face of increasing vaccination coverage. Finally, we have not accounted for outcomes of SARS-CoV-2 infection such as hospitalization and death, while the statistics of these two events often drive the lock downs and recommendation for physical distancing measures. However, we have provide the breakdown of the infections by the vaccination status (Figure 4). This figure can provide an intuition about the expected increase of the severity of epidemiological burden as new more infectious virus variants may emerge.

A number of studies/reports estimated the bounds for vaccine efficacy for the original variant in terms of reducing the infection for some vaccines approved for use in Europe [64, 65, 24, 25]. As Alpha (B.1.1.7) and Delta (B.1.617.2) variants emerged and, in turn, became dominant in many European countries, the first estimates for vaccine efficacy for reducing the infection became available $[31,32,66]$. Whether the reduction in infection comes in the guise of reduction of susceptibility or transmissibility of vaccinated individuals is not known. Therefore, in this work we modeled the vaccination to be all-or-nothing and vaccine efficacy was given in terms of probability of conferring full protection from becoming infected. Our sensitivity analyses (Figure 5 and Figures $\mathbf{1}, \mathbf{2}, \mathbf{4}$, and $\mathbf{5}$ in Supplementary materials) show that the effect of a vaccination campaign and of individual interventions is highly sensitive with respect to this parameter. However, we observed that if no compliance-targeting interventions accompany the vaccination rollout, the range of efficacies for which a surplus of new infections as compared to no-vaccination is possible three and six months following the vaccination rollout falls within the vaccine efficacy boundaries that were reported for different vaccines [31, 32, 65, 24, 25, 29, 27, 26, 67]. To implement the most efficient vaccination rollout it is important to know the boundaries of vaccine-conferred reduction of transmission.

Finally, in this work we have considered dynamics of circulation of three SARS-CoV-2 virus variants, the original variant and two mutations, whose transmission potential is similar to the Alpha and Delta variants. For all three variants, we modeled the immunity induced by the vaccine to be of the identical type (sterilising). 
Our results also show that speed of rollout of a vaccination campaign is important, because the speed of the rollout and subsequent changes in contact rates strongly impact cumulative number of new infections. Although given the scenario where vaccine efficacy is low and vaccination rollout is fast the population may observe a higher number of new infections than it would have been without vaccination in the short term - especially for a more transmissible virus variant - on the longer term (>1 year) it has vast advantages in terms of numbers of infections prevented.

Our results emphasize the importance of communication by public health professionals on continued adherence to self-imposed measures, to those who are awaiting vaccination as well as to those already vaccinated. Communication messages need to be different and targeted specifically to these two groups. We highlight the positive overall effects of vaccination campaigns in combination with continued adherence to non-pharmaceutical preventive measures.

\section{Methods}

\section{Model}

We developed a compartmental deterministic model that describes SARS-CoV-2 transmission and vaccination rollout in a population. Subsequently, we modified this model to include acquisition and loss of compliance with physical distancing measures as individuals continuously get exposed to information about disease spread as well as about the progress of vaccination rollout (Figure 2). We informed the model using parameter values from the literature as well as estimating parameters from publicly available data for the Netherlands, Belarus, Denmark, and Israel. We used the model to investigate the effects of interactions between disease transmission, vaccination rollout, and changing compliance with physical distancing measures on transmission dynamics.

Population compartments The SARS-CoV-2 transmission dynamics follow a SusceptibleExposed-Infectious-Recovered (SEIR) framework that divides the population into the following compartments: susceptible $(S)$, latently infected (also referred to as "exposed", $E$ ), infectious $(I)$, and recovered $(R)$. Susceptible individuals $(S)$ become latently infected $(E)$ with rate $\lambda_{\text {inf }}$ proportional to the fraction of infectious individuals $(I / N$, where $N$ is the total population size). Individuals stay latently infected $(E)$ for an average duration of $1 / \alpha$ days after which they become infectious $(I)$. Infectious individuals recover after $1 / \gamma$ days and move to compartment $R$. Because of a relatively short time horizon of our analyses (not exceeding six months) and relatively small case fatality ratio, we disregarded demographic processes such as births and deaths, and therefore the population size $N$ is constant. Additionally, we assumed that once individuals recover they acquire permanent immunity and cannot be re-infected. Since we are interested in understanding the qualitative dynamics that follow from interaction of infection transmission, changes in compliance, and vaccination rollout, we did not consider different outcomes of infection with SARS-CoV-2 (e.g., asymptomatic or symptomatic infection, hospitalisation, death etc.). The infectious compartment $(I)$, therefore, contains individuals who are asymptomatic, or have mild or severe symptoms.

The dynamics of infection transmission are modelled for three variants of the SARS-CoV-2 virus: first, the original variant that was predominant in Europe prior to fall 2020; second, the more transmissible Alpha (B.1.1.7) variant, that was initially detected in the UK and became dominant in many European countries in the spring of 2021; and finally, the "hyper-contagious" Delta (B.1.617.2) variant, which became dominant in Europe in summer 2021. We parameterized the differences between these variants by using different probabilities of transmission per contact, $\epsilon$. We assumed that in all other respects the variants have the same properties. We investigated 

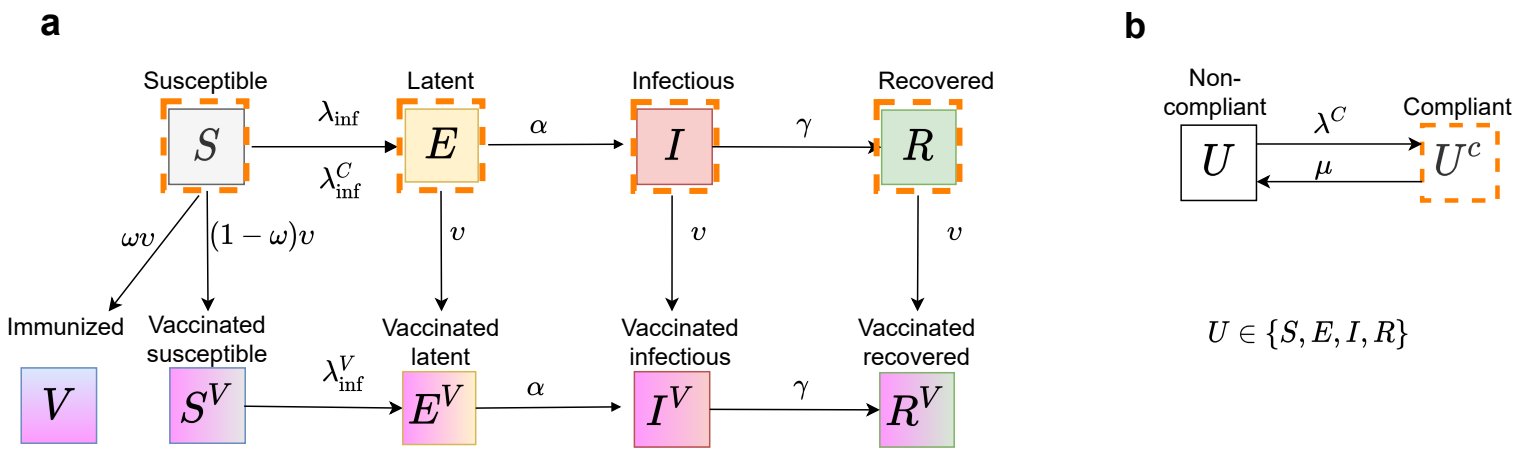

$U \in\{S, E, I, R\}$

Figure 2: Flow diagram of the infection transmission dynamics coupled with compliance and vaccination processes. a Flow diagram of infection transmission and vaccination rollout, b Flow diagram of acquisition and loss of compliance. Solid-colored rectangles denote nonvaccinated compartments; solid-bordered rectangles denote non-compliant compartments; orange dashed-bordered rectangles denote compliant compartments; gradient-colored rectangles denote vaccinated compartments. Susceptible individuals $\left(S, S^{C}\right.$, and $\left.S^{V}\right)$ become exposed $\left(E, E^{C}\right.$, and $E^{V}$, respectively) with rates $\lambda_{\text {inf }}, \lambda_{\text {inf }}^{C}$, and $\lambda_{\text {inf }}^{V}$ through contact with infectious individuals $\left(I, I^{C}\right.$, and $\left.I^{V}\right)$. Exposed individuals become infectious $\left(I, I^{C}\right.$, and $I^{V}$, respectively) at rate $\alpha$. Infectious individuals recover $\left(R, R^{C}\right.$, and $\left.R^{V}\right)$ at rate $\gamma$. Compliance is gained with rate $\lambda^{C}$ and lost with rate $\mu$. Individuals in any state of infection or compliance can get vaccinated. A proportion $\omega$ of susceptible individuals $S$, who were vaccinated are fully protected, $V$. Individuals who were vaccinated, but did not obtain protection, are denoted by $S^{V}, E^{V}, I^{V}$ and $R^{V}$ and are epidemiologically indistinguishable from their non-vaccinated counterparts.

model dynamics where only one of the three variants circulates in the population.

To model vaccination, the population was stratified into vaccinated and non-vaccinated classes. While for some vaccines authorised for use in Europe (BioNTech/Pfizer, Moderna and AstraZeneca, [18]), two vaccine doses, as well as a certain time period passing after the second dose are required for full immunisation, we modelled vaccination as a single event that confers protection instantaneously. We assumed that individuals do not obtain a diagnostic or antibody test prior to vaccination, and therefore infected and recovered individuals also get vaccinated. Thus, individuals in all epidemiological compartments can get vaccinated, but only those who were susceptible $(S)$ at the time of vaccination may become immunised $(V)$. The vaccination rate is denoted by $v$. We assumed that the vaccine works as all-or-nothing, i.e. upon vaccination, a proportion $\omega$ of susceptible individuals $(S)$ is fully protected $(V)$, while in a proportion $1-\omega$ of susceptible individuals the vaccine has no effect. We refer to $\omega$ as "vaccine efficacy" in the context of conferring sterilising immunity. Vaccination does not confer protection to individuals, who were in other infection compartments $(E, I$ and $R$ ) at the time of vaccination, and their infection progression is identical to that of non-vaccinated individuals. Individuals who were vaccinated but did not obtain the protection are denoted by $S^{V}, E^{V}, I^{V}$ and $R^{V}$.

Studies based on data collected in Israel estimated that the BNT162b2 mRNA COVID-19 vaccine developed by Pfizer/BioNTech reduced the acquisition rate for asymptomatic SARS-CoV2 infection by $80 \%$ [26] up to 95\% [27]. Similarly high efficacy against infection acquisition were reported for the mRNA-1273 COVID-19 vaccine developed by Moderna, NIAID [28]. For the adenovirus Ad26.COV2.S COVID-19 vaccine developed by Janssen Pharmaceutical Companies the efficacy in preventing infection with SARS-CoV-2 is reported to be $76 \%$ [29]. These results were estimated from data collected between December 2020 and April 2021 in the USA. During 
this period, the original variant and the Alpha variant (B.1.1.7) were the dominant circulating variants. Since then, the "hyper-contagious" Delta variant (B.1.617.2) has become dominant in many European countries [10] and the USA [30]. A recent study based on data from Israel estimated a significant reduction of BNT162b2 efficacy for the Delta variant in preventing infection, which was $64 \%$ after two doses [31]. This estimate was supported by another report based on the data in a highly vaccinated health system workforce of California San Diego Health [32]. Therefore, in our analyses, we varied $\omega$ in the range of 0.4 and 1.0 .

Finally, in addition to infection status, unvaccinated individuals in the model are either compliant or non-compliant with physical distancing measures (compliant compartments denoted by superscript $C: S^{C}, E^{C}, I^{C}$, and $R^{C}$ ). Compliant individuals thus have on average a lower contact rate than non-compliant individuals; both contact rates are assumed to be lower than pre-pandemic levels. We denote the contact rate of non-compliant individuals by $c$, and define a reduction factor $r_{1}$ that describes the reduction in contact rate of compliant individuals compared to non-compliant individuals, so $0 \leq r_{1} \leq 1$. Transitions between the compliant and non-compliant state are described by a modelling framework similar to Perra et al [46] and previously used in [48]. We modeled the compliance acquisition rate, $\lambda_{C}$, as a function of the incidence of infection, assuming that individuals obtain information about numbers of cases through mass-media and health authorities. We assumed that compliance wanes when case numbers drop or when the disease is no longer present, and individuals return to the non-compliant state at rate $\mu$. If there is no vaccination programme in place then this rate, $\mu$, is constant. However, if vaccination rollout is in progress and as vaccination coverage increases, individuals may feel less motivated to comply with physical distancing measures; we implemented this effect by taking $\mu$ as a linear function of vaccination coverage, i.e. the rate of losing compliance increases with increasing vaccination coverage. We assumed that only non-vaccinated individuals can be in the compliant state, while vaccinated individuals move into a separate non-compliant state permanently, and even have higher contact rates than non-vaccinated non-compliant individuals. We use $r_{2} \geq 1$ to denote the increase in the contact rates of vaccinated individuals relative to the contact rate of non-compliant individuals, $c$. Compliant individuals get vaccinated at the same rate as non-compliant individuals. All individuals who were vaccinated will have the same (increased) contact rate regardless of whether vaccination was successful.

Rates In this section we define the transition rates that depend on the incidence of infectious cases and on vaccination coverage: rates of infection acquisition, and rates of acquisition and loss of compliance.

We assumed that individuals become infected at a rate that depends on the fractions of different types of infectious individuals, as well as on the mixing of compliant, non-compliant and vaccinated individuals. Therefore, infection acquisition rates as well as infection transmission rates depend on compliance and vaccination status of susceptible and infectious individuals. We define the following matrix to summarize transmission rates between different types of susceptible and infectious individuals.

$$
M=\frac{c \epsilon}{N(t)+r_{1} N^{C}(t)+r_{2} N^{V}(t)}\left[\begin{array}{ccc}
1 & r_{1} & r_{2} \\
r_{1} & r_{1}^{2} & r_{1} r_{2} \\
r_{2} & r_{1} r_{2} & r_{2}^{2}
\end{array}\right]
$$


with

$$
\begin{aligned}
N(t) & =S(t)+E(t)+I(t)+R(t) \\
N^{C}(t) & =S^{C}(t)+E^{C}(t)+I^{C}(t)+R^{C}(t) \\
N^{V}(t) & =V(t)+S^{V}+E^{V}(t)+I^{V}(t)+R^{V}(t),
\end{aligned}
$$

where $[M]_{11}$ captures the transmission of infection from non-compliant $I$ to non-compliant $S,[M]_{12}$ from compliant $I$ to non-compliant $S$, and $[M]_{13}$ from vaccinated $I$ to non-compliant $S$. Similarly, the second row of the matrix captures the transmission of infection to susceptible individuals who are compliant, $S^{C}$. Finally, the third row of the matrix captures the transmission of infection to individuals who are susceptible despite vaccination, $S^{V}$.

We assumed that as individuals learn about new infections they become compliant with physical distancing measures, and therefore compliance is gained at a rate $\lambda_{C}$ which is a positive increasing function of the incidence of infectious cases (equal to the rate with which individuals leave the exposed stage):

$$
\lambda_{\mathrm{C}}(t)=\delta \cdot \alpha \cdot\left[E(t)+E^{C}(t)+E^{V}(t)\right] .
$$

We assumed that compliance is not permanent, becoming shorter as the vaccination coverage grows, and thus we model compliant state to have an average duration $1 / \mu$, such that $\mu$ is a positive increasing function of the vaccination coverage, $\bar{V}(t) / N$ :

$$
\mu(t)=\mu_{0}+\mu_{1} \bar{V}(t) / N .
$$

Equations The system of ordinary differential equations (4) provides a full description of the model.

Dynamics of non-compliant individuals:

$$
\begin{aligned}
\frac{\mathrm{d} S(t)}{\mathrm{d} t}= & -\lambda_{\text {inf }}(t) S(t)-\lambda_{\mathrm{C}}(t) S(t)+\mu(t) S^{C}(t)-v S(t) \\
\frac{\mathrm{d} E(t)}{\mathrm{d} t}= & \lambda_{\text {inf }}(t) S(t)-\alpha E(t)-\lambda_{\mathrm{C}}(t) E(t)+\mu(t) E^{C}(t) \\
& -v E(t) \\
\frac{\mathrm{d} I(t)}{\mathrm{d} t}= & \alpha E(t)-\gamma I(t)-\lambda_{\mathrm{C}}(t) I(t)+\mu(t) I^{C}(t)-v I(t) \\
\frac{\mathrm{d} R(t)}{\mathrm{d} t}= & \gamma I(t)-\lambda_{\mathrm{C}}(t)+R(t) \mu(t) R^{C}(t)-v R(t)
\end{aligned}
$$

Dynamics of compliant individuals

$$
\begin{aligned}
\frac{\mathrm{d} S^{C}(t)}{\mathrm{d} t}= & -\lambda_{\text {inf }}^{C}(t) S^{C}(t)+\lambda_{\mathrm{C}}(t) S(t)-\mu(t) S^{C}(t)-v S^{C}(t) \\
\frac{\mathrm{d} E^{C}(t)}{\mathrm{d} t}= & \lambda_{\text {inf }}^{C}(t) S^{C}(t)-\alpha E^{C}(t)+\lambda_{\mathrm{C}}(t) E(t)-\mu(t) E^{C}(t) \\
& -v E^{C}(t) \\
\frac{\mathrm{d} I^{C}(t)}{\mathrm{d} t}= & \alpha E^{C}(t)-\gamma I^{C}(t)+\lambda_{\mathrm{C}}(t) I(t)-\mu(t) I^{C}(t) \\
& -v I^{C}(t) \\
\frac{\mathrm{d} R^{C}(t)}{\mathrm{d} t}= & \gamma I^{C}(t)+\lambda_{\mathrm{C}}(t) R(t)-\mu(t) R^{C}(t)-v R^{C}(t)
\end{aligned}
$$


Dynamics of vaccinated individuals:

$$
\begin{aligned}
& \frac{\mathrm{d} V(t)}{\mathrm{d} t}=\omega v\left(S(t)+S^{C}(t)\right) \\
& \frac{\mathrm{d} S^{V}(t)}{\mathrm{d} t}=(1-\omega) v\left(S(t)+S^{C}(t)\right)-\lambda_{\text {inf }}^{V}(t) S^{V}(t) \\
& \frac{\mathrm{d} E^{V}(t)}{\mathrm{d} t}=\lambda_{\text {inf }}^{V}(t) S^{V}(t)+v\left(E(t)+E^{C}(t)\right)-\alpha E^{V}(t) \\
& \frac{\mathrm{d} I^{V}(t)}{\mathrm{d} t}=\alpha E^{V}(t)+v\left(I(t)+I^{C}(t)\right)-\gamma I^{V}(t) \\
& \frac{\mathrm{d} R^{V}(t)}{\mathrm{d} t}=\gamma I^{V}(t)+v\left(R(t)+R^{C}(t)\right) \\
& \frac{\mathrm{d} \bar{V}(t)}{\mathrm{d} t}= v\left(S(t)+E(t)+R(t)+S^{C}(t)+E^{C}(t)+R^{C}(t)\right) \\
&+v\left(I(t)+I^{C}(t)\right),
\end{aligned}
$$

where

$$
\begin{aligned}
& \lambda_{\mathrm{inf}}(t)=[M(t)]_{11} I(t)+[M(t)]_{12} I^{C}(t)+[M(t)]_{13} I^{V}(t) \\
& \lambda_{\mathrm{inf}}^{C}(t)=[M(t)]_{21} I(t)+[M(t)]_{22} I^{C}(t)+[M(t)]_{23} I^{V}(t) \\
& \lambda_{\mathrm{inf}}^{V}(t)=[M(t)]_{31} I(t)+[M(t)]_{32} I^{C}(t)+[M(t)]_{33} I^{V}(t) .
\end{aligned}
$$

\section{Parameters and initial data}

A full list of parameters and their values is given in Table 1. Here we elaborate on our choice of initial conditions, as well as on the chosen values of the behavioral parameters.

Initial data To model the dynamics of SARS-CoV-2 we used the approximation made by RIVM for the week November 11-17 for the number of infectious individuals and set the total number of currently infectious individuals, $I+I^{C}$, at the start of vaccination rollout to 112,435 [4]. We have used this value in the main analysis and performed sensitivity analysis to investigate the sensitivity of our results to this choice. To estimate the fraction of recovered individuals, $R+R_{C}$ we used seroprevalence data from a serological study conducted in the Netherlands in June and July of 2020. We set the number of recovered, $R+R^{C}$ such that at the start of vaccination rollout, which was in January 2021 in the Netherlands, the fraction of recovered in the population was $8 \%$, and performed sensitivity analysis with respect to this initial value.

To estimate the total number of exposed individuals $E+E^{C}$ at the start of the vaccination rollout, we assumed that, at the time, the epidemiological dynamics are in (pseudo) equilibrium, with the prevalence of infectious cases equal to 112,435 individuals [4]. Using the average duration of infectious period equal to 7 days [72], we estimated that, at the start of the vaccination rollout, the daily incidence of new cases was 16,062 individuals. Using the average duration of the exposed period of infection equal to 4 days [68, 69, 71], we obtained $E+E^{C}$. Having fixed the size of susceptible $\left(S+S^{C}\right)$, exposed $\left(E+E^{C}\right)$, and recovered $\left(R+R^{C}\right)$ compartments and using the total population size of the Netherlands, the size of the susceptible compartment $\left(S+S^{C}\right)$ follows.

We have set the initial proportion of compliant individuals to $65 \%$. This was based on data on the compliance with maintaining a distance of $1.5 \mathrm{~m}$, from a study on behavioral measures and well-being conducted between November 11-15, 2020 [53] in the Netherlands. 
Table 1: Summary of model parameters.

\begin{tabular}{|c|c|c|c|}
\hline Name & Description (unit) & Value* & Source \\
\hline \multicolumn{4}{|c|}{ Epidemiological parameters } \\
\hline$R_{0}$ & Basic reproduction number, original variant & 2.5 & {$[68,69]$} \\
\hline$R_{0}^{\text {new }}$ & $\begin{array}{l}\text { Basic reproduction number, Alpha (B.1.1.7)- } \\
\text { like variant }\end{array}$ & 3.75 & {$[6,7]$} \\
\hline$R_{0}^{\text {new }}$ & $\begin{array}{l}\text { Basic reproduction number, } \\
\text { (B.1.617.2)-like variant }\end{array}$ & 4.92 & {$[55]$} \\
\hline$R_{e}$ & $\begin{array}{l}\text { Effective reproduction number, original vari- } \\
\text { ant }\end{array}$ & 1.1 & $\begin{array}{l}\text { Computed using the } \\
\text { method in [70] }\end{array}$ \\
\hline$\hat{c}$ & $\begin{array}{l}\text { Average contact rate prior to the epidemic } \\
\text { (individuals/day) }\end{array}$ & 14.9 & {$[56]$} \\
\hline$\epsilon$ & $\begin{array}{l}\text { Probability of transmission per contact, orig- } \\
\text { inal variant }\end{array}$ & $2.4 \times 10^{-2}$ & From $R_{0}=\hat{c} \epsilon / \gamma=2.5$ \\
\hline$\epsilon^{\text {Alpha }}$ & $\begin{array}{l}\text { Probability of transmission per contact, } \\
\text { Alpha-like variant }\end{array}$ & $3.6 \times 10^{-2}$ & $\begin{array}{l}\text { From } R_{0}=\hat{c} \epsilon^{A l p h a} / \gamma= \\
3.75\end{array}$ \\
\hline$\epsilon^{\text {Delta }}$ & $\begin{array}{l}\text { Probability of transmission per contact, } \\
\text { Delta-like variant }\end{array}$ & $5.4 \times 10^{-2}$ & $\begin{array}{l}\text { From } R_{0}=\hat{c} \epsilon^{\text {Delta }} / \gamma= \\
4.92\end{array}$ \\
\hline$c$ & $\begin{array}{l}\text { Average contact rate of non-compliant indi- } \\
\text { viduals starting November 16, } 2020 \text { (individ- } \\
\text { uals/day) }\end{array}$ & $8.8(0.5-15)$ & $\begin{array}{l}\text { Obtained from solving } \\
R_{e}(0)=1.1\end{array}$ \\
\hline$r_{1}$ & $\begin{array}{l}\text { Ratio between contact rates of compliant and } \\
\text { non-compliant individuals }\end{array}$ & $0.34(0.01-1)$ & $\begin{array}{l}\text { Assumed, control param- } \\
\text { eter }\end{array}$ \\
\hline$r_{2}$ & $\begin{array}{l}\text { Ratio between contact rates of vaccinated } \\
\text { and non-compliant individuals }\end{array}$ & $1.5(1,1.5)$ & $\begin{array}{l}\text { Assumed, control param- } \\
\text { eter }\end{array}$ \\
\hline $1 / \alpha$ & $\begin{array}{l}\text { Duration of latent period } \\
\text { (days) }\end{array}$ & $4(2-6)$ & {$[68,69,71]$} \\
\hline $1 / \gamma$ & Duration of infectious period (days) & $7(5-9)$ & {$[72]$} \\
\hline \multicolumn{4}{|c|}{ Compliance parameters } \\
\hline$\delta$ & Rate of moving to compliant state (1/day) & $\begin{array}{l}4 \times 10^{-5}\left(10^{-6}-\right. \\
\left.10^{-4}\right)\end{array}$ & $\begin{array}{l}\text { Assumed, control param- } \\
\text { eter }\end{array}$ \\
\hline $1 / \mu_{0}$ & $\begin{array}{l}\text { Duration of compliant state when there is no } \\
\text { vaccination (days) }\end{array}$ & $30(7-30)$ & Sensitivity analyses \\
\hline$\mu_{1}$ & $\begin{array}{l}\text { Parameter describing how loss of compliance } \\
\text { increases depending on vaccination coverage } \\
\text { (1/day) }\end{array}$ & $0,0.3$ & Sensitivity analyses \\
\hline \multicolumn{4}{|c|}{ Vaccination parameters } \\
\hline$v$ & Vaccine uptake rate (1/day) & $(5,60) \times 10^{-3}$ & $\begin{array}{l}\text { Based on vaccination } \\
\text { data in [39] }\end{array}$ \\
\hline$\omega$ & $\begin{array}{l}\text { Vaccine efficacy in conferring protection } \\
\text { against becoming infected }\end{array}$ & $0.6(0.55-0.95)$ & $\begin{array}{l}{[32,65,24,25,29,31,27,} \\
26,67], \text { control parame- } \\
\text { ter }\end{array}$ \\
\hline \multicolumn{4}{|c|}{ Lockdown parameters } \\
\hline & $\begin{array}{l}\text { Threshold of infectious individuals for } \\
\text { strengthening/relaxation of the lockdown } \\
\text { (individuals) }\end{array}$ & $50-500$ & Sensitivity analysis \\
\hline & $\begin{array}{l}\text { Average contact rate during strengthened } \\
\text { lockdown (individuals/day) }\end{array}$ & 3 & Sensitivity analysis \\
\hline
\end{tabular}

* Interval was used in sensitivity analyses. 
We obtain

$$
\frac{S}{S+S^{C}}=\frac{E}{E+E^{C}}=\frac{I}{I+I^{C}}=\frac{R}{R+R^{C}}
$$

Using Eq. (6) and the percentage of compliant population, initial values for $S, E, I, R, S^{C}, E^{C}$, $I^{C}, R^{C}$ follow.

Setting the total population size to be equal to approximately that of the Netherlands, $1.7 \times 10^{7}$ we obtain the initial data:

$$
\begin{aligned}
& S(0)=5,412,160, E(0)=22,487, I(0)=39,352, \\
& R(0)=476,000, S^{C}(0)=10,051,156, E^{C}(0)=41,762 \\
& I^{C}(0)=73,082, R^{C}(0)=884,000 .
\end{aligned}
$$

The initial values for the remaining compartments are set to 0 .

Contact rates We defined a contact as an encounter with another individual that is sufficiently long to have a conversation, or that involves physical interactions [56]. The pre-pandemic contact rate in the Netherlands was reported to be equal to 14.9 individuals per day [56]. We assume that the population is in the state of a partial lockdown at the start and throughout the vaccination rollout. In addition to the lockdown-related changes in the contact rate, individuals may reduce the contact rate further by complying with government-recommended physical distancing measures (e.g. work from home as much as possible). A fraction of the population is more compliant with these physical distancing measures and the remaining fraction is less compliant, such that contact rates in the compliant and non-compliant states are constant and the average contact rate is lower than pre-pandemic contact rate. However, as a consequence of vaccination and subsequent loss of compliance the average contact rate in the total population will change in time.

We fixed the contact rates for compliant and non-compliant individuals such that the effective reproduction number $R_{e}$ at the start of the vaccination rollout was 1.1, which is in agreement with the estimate of $R_{t}$ reported for the Netherlands in November 2020 [54]. We calculated $R_{e}(0)$ assuming that $R_{0}=\beta / \gamma=\hat{c} \epsilon / \gamma=2.5[68,69]$.

Recall that the contact rates of non-compliant and compliant individuals are denoted by $c$ and $r_{1} c$. We calculated the effective reproduction number using the method described in [70] as

$$
R_{e}=\frac{\epsilon c S(0)}{\gamma\left(N(0)+N^{c}(0) r_{1}\right)}+\frac{\epsilon r_{1} c S_{c}(0)\left(\mu_{0}\left(\alpha+\gamma+\mu_{0}\right)+\alpha \gamma r_{1}\right)}{\gamma\left(\alpha+\mu_{0}\right)\left(\gamma+\mu_{0}\right)\left(N(0)+N^{c}(0) r_{1}\right)} .
$$

The value $R_{e}=1.1$ is obtained for pairs of contact rates of non-compliant individuals, $c$, and compliant individuals, $r_{1} c$ (see Supplementary materials, Section 4, Figure 17).

Of all pairs of contact rates that satisfy $R_{e}(0)=1.1$, we selected a combination such that the weighted average contact rate for the population at the start of the vaccination is 5 contacts per day. This value exceeds the reported number of contacts in the Netherlands during the governmentimposed physical distancing measures in March 2020 by 1.5 contacts but is lower than the reported contact rate of 8.8 per day that was observed in June 2020, when some of the physical distancing measures were relaxed [56]. The chosen parameter pair is $c=8.8$ and $r_{1} c=2.8$.

Contact rates of vaccinated individuals were taken to be 1.5 times the contact rate of noncompliant individuals, assuming that after vaccination individuals will nearly return to the prepandemic contact behaviour.

Compliance The proportion of compliant and non-compliant individuals in the population is determined by the compliance acquisition rate $\delta$ and compliance loss rate $\mu$. For the main analysis 
we fixed the duration of compliance when there is no vaccination, $1 / \mu_{0}$ to 30 days. We selected the per capita rate of moving to the compliant state, $\delta=4 \times 10^{-5}$ so that given a constant daily incidence of 16,062 cases, $95 \%$ of the population is expected to be compliant. In the regime where the epidemic is seeded with the original variant in a population without any physical measures as much as $84 \%$ of the population can be compliant provided there were no compliant individuals at the start of the epidemic. This value denotes the case with high compliance acquisition rate. We investigated the sensitivity of the outputs to variation in per capita rate of moving to the compliant state and the compliance loss rate (Supplementary materials).

In the main analysis we considered a compliance decay scenario where as the vaccination coverage grows, the duration of compliance decreases, in particular when $33 \%$ of the population is vaccinated the compliant state lasts on average 7 days. In other words, for a daily incidence of 16,062 cases, which we used to initialize the model, and a slow vaccination rate, approximately $83 \%$ of the population is still compliant 6 months after start of the vaccination rollout, while for a fast vaccination rate only $32 \%$ are compliant (Figure $2 \mathbf{b}$ ). These dynamics occur when the growth rate of compliant decay rate as the vaccination coverage increase is $\mu_{1}=0.3$ per day.

Model code The model was implemented in MATLAB R2020b [73]. The code producing the analyses and figures for this study is available at https://github.com/aiteslya/VaccineCompliance1.2 [74].

Acknowledgments We thank Marc Bonten (UMC Utrecht) for comments on an earlier version of the manuscript. MEK acknowledges support from the Netherlands Organization for Health Research and Development (ZonMw; Grant no. 91216062 and Grant no. 10430022010001). GR acknowledges support from the Portuguese Foundation for Science and Technology (FCT; Grant no. 131_596787873). AT and HN acknowledge support from the Netherlands Organization for Health Research and Development (ZonMw; Grant no. 91216062).

\section{References}

[1] World Health Organization. WHO Director-General's opening remarks at the media briefing on COVID-19 - 11 March 2020, 2020. URL https://www.who.int/director-general/speeches/detail/ who-director-general-s-opening-remarks-at-the-media-briefing-on-covid-19---11-march-2 Accessed February 25, 2021.

[2] World Health Organization. WHO Coronavirus (COVID-19) Dashboard, 2021. URL https: //covid19.who.int. Accessed April 2, 2021.

[3] M-K Looi. Covid-19: Is a second wave hitting Europe? The BMJ, 371, 2020. doi: 10.1136/ bmj.m4113. URL https://www.bmj.com/content/371/bmj.m4113.

[4] Number of infectious people: Coronavirus Dashboard:Government.nl, 2021. URL https: //coronadashboard.government.nl/landelijk/besmettelijke-mensen. Accessed August 4, 2021.

[5] European Centre for Disease Prevention and Control. Rapid increase of a SARSCoV-2 variant with multiple spike protein mutations observed in the United Kingdom, 2020. URL https://www.ecdc.europa.eu/en/publications-data/ threat-assessment-brief-rapid-increase-sars-cov-2-variant-united-kingdom. Accessed March 4, 2021. 
[6] NG Davies, S Abbott, RC Barnard, CI Jarvis, AJ Kucharski, JD Munday, et al. Estimated transmissibility and impact of SARS-CoV-2 lineage B.1.1.7 in England. Science, 2021. doi: 10.1126/science.abg3055. URL https://science.sciencemag.org/content/early/2021/ 03/03/science. abg3055.

[7] E Volz, S Mishra, M Chand, JC Barrett, R Johnson, L Geidelberg, et al. Transmission of SARS-CoV-2 Lineage B.1.1.7 in England: Insights from linking epidemiological and genetic data. medRxiv, 2021. doi: 10.1101/2020.12.30.20249034. URL https://www.medrxiv.org/ content/early/2021/01/04/2020.12.30.20249034.1.

[8] NG Davies, CI Jarvis, CMMID COVID-19 Working Group, et al. Increased mortality in community-tested cases of SARS-CoV-2 lineage B.1.1.7. Nature, 2021. doi: 10.1038/ s41586-021-03426-1. URL https://www.nature.com/articles/s41586-021-03426-1.

[9] R Challen, E Brooks-Pollock, JM Read, L Dyson, K Tsaneva-Atanasova, and L Danon. Risk of mortality in patients infected with SARS-CoV-2 variant of concern 202012/1: matched cohort study. The BMJ, 372, 2021. doi: 10.1136/bmj.n579. URL https://www.bmj.com/ content/372/bmj.n579.

[10] WHO/Europe: SARS-CoV-2 Delta variant now dominant in much of European region; efforts must be reinforced to prevent transmission, warns WHO Regional Office for Europe and ECDC, 2021. URL https: //www. euro.who.int/en/media-centre/sections/press-releases/2021/ sars-cov-2-delta-variant-now-dominant-in-much-of-european-region-efforts-must-be-rein -warns-who-regional-office-for-europe-and-ecdc. Accessed September 6, 2021.

[11] Centers for Disease Control and Prevention: CDC COVID Data Tracker, 2021. URL https:// covid.cdc.gov/covid-data-tracker/\#variant-proportions. Accessed January 29, 2022.

[12] Our World In Data. SARS-CoV-2 sequences by variant, Jan 28, 2022, 2022. URL https://ourworldindata.org/grapher/covid-variants-bar?country= $\mathrm{AUS} \sim \mathrm{GBR} \sim \mathrm{USA} \sim \mathrm{BEL} \sim \mathrm{ITA} \sim \mathrm{FRA} \sim \mathrm{ESP} \sim \mathrm{DEU} \sim \mathrm{BWA} \sim \mathrm{ZAF} \sim \mathrm{CAN}$. Accessed January 29, 2022.

[13] H Lau, V Khosrawipour, P Kocbach, A Mikolajczyk, J Schubert, J Bania, et al. The positive impact of lockdown in Wuhan on containing the COVID-19 outbreak in China. Journal of Travel Medicine, 27(3), 2020. doi: 10.1093/jtm/taaa037. URL https://pubmed.ncbi.nlm. nih.gov/32181488/.

[14] T VoPham, MD Weaver, JE Hart, M Ton, E White, and PA Newcomb. Effect of social distancing on COVID-19 incidence and mortality in the US. MedRxiv, 2020. doi: 10.1101/ 2020.06.10.20127589. URL https://www.ncbi.nlm.nih.gov/pmc/articles/PMC7310657/.

[15] JM Brauner, S Mindermann, M Sharma, D Johnston, J Salvatier, T Gavenčiak, et al. Inferring the effectiveness of government interventions against COVID-19. Science, 371(6531), 2021. doi: 10.1126/science.abd9338. URL https://science.sciencemag.org/content/ $371 / 6531 /$ eabd9338.

[16] Y Li, H Campbell, D Kulkarni, A Harpur, M Nundy, PX Wang, et al. The temporal association of introducing and lifting non-pharmaceutical interventions with the time-varying reproduction number (R) of SARS-CoV-2: a modelling study across 131 countries. The Lancet Infec- 
tious Diseases, 21(2):193 - 202, 2021. doi: 10.1016/S1473-3099(20)30785-4. URL https:// www. thelancet.com/journals/laninf/article/PIIS1473-3099(20)30785-4/fulltext.

[17] Y Liu, C Morgenstern, J Kelly, R Lowe, CMMID COVID-19 Working Group, and M Jit. The impact of non-pharmaceutical interventions on SARS-CoV-2 transmission across 130 countries and territories. BMC Medicine, 19, 2021. doi: 10.1186/s12916-020-01872-8. URL https://bmcmedicine.biomedcentral.com/articles/10.1186/s12916-020-01872-8.

[18] European Medicines Agency. COVID-19 vaccines: authorised, 2021. URL https://europa. eu/! bf 46fd. Accessed March 4, 2021.

[19] Fda: Covid-19 vaccines, 2022. URL https://www.fda.gov/ emergency-preparedness-and-response/coronavirus-disease-2019-covid-19/ covid-19-vaccines\#authorized-vaccines. Accessed January 29, 2022.

[20] FP Polack, SJ Thomas, N Kitchin, J Absalon, A Gurtman, S Lockhart, et al. Safety and efficacy of the BNT162b2 mRNA Covid-19 vaccine. New England Journal of Medicine, 383 (27):2603-2615, 2020. doi: 10.1056/NEJMoa2034577. URL https://doi.org/10.1056/ NEJMoa2034577.

[21] LR Baden, HM El Sahly, B Essink, K Kotloff, S Frey, R Novak, et al. Efficacy and safety of the mRNA-1273 SARS-CoV-2 vaccine. New England Journal of Medicine, 384(5):403-416, 2021. doi: 10.1056/NEJMoa2035389. URL https://www.nejm.org/doi/full/10.1056/ nejmoa2035389.

[22] M Voysey, SAC Clemens, SA Madhi, LY Weckx, PM Folegatti, Aley PK, et al. Safety and efficacy of the ChAdOx1 nCoV-19 vaccine (AZD1222) against SARS-CoV-2: an interim analysis of four randomised controlled trials in Brazil, South Africa, and the UK. The Lancet, 397:99-111, 2020. doi: 10.1016/S0140-6736(20)32661-1. URL https://www. thelancet.com/ journals/lancet/article/PIIS0140-6736(20)32661-1/fulltext.

[23] Real-World Evidence Confirms High Effectiveness of Pfizer-BioNTech COVID-19 Vaccine and Profound Public Health Impact of Vaccination One Year After Pandemic Declared, 2021. URL https://www. businesswire.com/news/home/20210311005482/en/. Accessed March $16,2021$.

[24] Marc Lipsitch and Rebecca Kahn. Interpreting vaccine efficacy trial results for infection and transmission. Vaccine, 39(30):4082-4088, 2021. ISSN 0264-410X. doi: https://doi.org/ 10.1016/j.vaccine.2021.06.011. URL https://www.sciencedirect.com/science/article/ $\mathrm{pii} / \mathrm{S} 0264410 \mathrm{X} 21007350$.

[25] ASV Shah, C Gribben, J Bishop, P Hanlon, D Caldwell, R Wood, et al. Effect of vaccination on transmission of COVID-19: an observational study in healthcare workers and their households. medRxiv, 2021. doi: 10.1101/2021.03.11.21253275. URL https: //www.medrxiv.org/content/early/2021/03/21/2021.03.11.21253275.

[26] O Prunas, JL Warren, FW Crawford, S Gazit, T Patalon, DM Weinberger, and VE Pitzer. Vaccination with BNT162b2 reduces transmission of SARS-CoV-2 to household contacts in Israel. medRxiv, 2021. doi: 10.1101/2021.07.13.21260393. URL https://www.medrxiv.org/ content/early/2021/07/16/2021.07.13.21260393. 
[27] EJ Haas, FJ Angulo, JM McLaughlin, E Anis, SR Singer, F Khan, et al. Impact and effectiveness of mRNA BNT162b2 vaccine against SARS-CoV-2 infections and COVID-19 cases, hospitalisations, and deaths following a nationwide vaccination campaign in Israel: an observational study using national surveillance data. The Lancet, 397:1819-1829, 2021. doi: 10.1016/S0140-6736(21)00947-8.

[28] C Pawlowski, P Lenehan, A Puranik, V Agarwal, AJ Venkatakrishnan, MJM Niesen, et al. FDA-authorized mRNA COVID-19 vaccines are effective per real-world evidence synthesized across a multi-state health system. Med, 2, 2021. doi: 10.1016/j.medj.2021.06.007.

[29] J Corchado-Garcia, D Puyraimond-Zemmour, T Hughes, T Cristea-Platon, P Lenehan, C Pawlowski, et al. Real-world effectiveness of Ad26.COV2.S adenoviral vector vaccine for COVID-19. medRxiv, 2021. doi: 10.1101/2021.04.27.21256193. URL https://www .medrxiv . org/content/early/2021/04/30/2021.04.27.21256193.

[30] Centers for Disease Control and Prevention: Delta Variant: What We Know About the Science: CDC, 2021. URL https://www.cdc.gov/coronavirus/2019-ncov/variants/ delta-variant.html. Accessed May 5, 2021.

[31] Decline in Vaccine Effectiveness Against Infection and Symptomatic Illness: Ministry of Health, 2021. URL https://www.gov.il/en/departments/news/05072021-03. Accessed August 11, 2021.

[32] J Keehner, LE Horton, NJ Binkin, LC Laurent, D Pride, CA Longhurst, et al. Resurgence of SARS-CoV-2 infection in a highly vaccinated health system workforce. New England Journal of Medicine, 2021. doi: 10.1056/NEJMc2112981. URL https://doi.org/10.1056/ NEJMc2112981.

[33] European Centre for Disease Prevention and Control. Risk related to the spread of new SARS-CoV-2 variants of concern in the EU/EEA - first update, 2021. URL https://www.ecdc.europa.eu/sites/default/files/documents/ COVID-19-risk-related-to-spread-of-new-SARS-CoV-2-variants-EU-EEA-first-update . pdf. Accessed March 9, 2021.

[34] D Planas, T Bruel, L Grzelak, F Guivel-Benhassine, I Staropoli, F Porro, et al. Sensitivity of infectious SARS-CoV-2 B.1.1.7 and B.1.351 variants to neutralizing antibodies. $\mathrm{Na}$ ture Medicine, 2021. doi: 10.1038/s41591-021-01318-5. URL https://www.nature.com/ articles/s41591-021-01318-5.

[35] Threat Assessment Brief: Emergence of SARS-CoV-2 B.1.617 variants in India and situation in the EU/EEA, 2021. URL https://www.ecdc.europa.eu/en/publications-data/ threat-assessment-emergence-sars-cov-2-b1617-variants. Accessed May 13, 2021.

[36] MC Mills and D Salisbury. The challenges of distributing COVID-19 vaccinations. EClinicalMedicine, 31, 2020. doi: 10.1016/j.eclinm.2020.100674. URL https://linkinghub. elsevier.com/retrieve/pii/S2589537020304181.

[37] AH Mokhad, PJ Hotez, and WA Orenstein. We have to get it right: Ensuring success. EClinicalMedicine, 31, 2020. doi: 10.1016/j.eclinm.2020.100690. URL https://www.thelancet. com/journals/eclinm/article/PIIS2589-5370(20)30434-X/fulltext. 
[38] J Wang, Y Peng, $\mathrm{H} \mathrm{Xu,} \mathrm{Z} \mathrm{Cui,} \mathrm{and} \mathrm{RO} \mathrm{Williams.} \mathrm{The} \mathrm{COVID-19} \mathrm{vaccine} \mathrm{race:} \mathrm{challenges}$ and opportunities in vaccine formulation. AAPS PharmSciTech, 21(6):1-12, aug 2020. ISSN 15309932. doi: 10.1208/s12249-020-01744-7. URL https://link.springer.com/article/ $10.1208 / \mathrm{s} 12249-020-01744-7$.

[39] Our World In Data. Coronavirus (COVID-19) Vaccinations, 2021. URL https:// ourworldindata.org/covid-vaccinations. Accessed August 3, 2021.

[40] European Centre for Disease Prevention and Control (ECDC) and the RECOVER Social Sciences team. Public views of COVID-19 vaccination in seven European countries: options for response, 2021. URL https://www.recover-europe.eu/wp-content/uploads/2021/ 02/Social-science-policy-brief_Vaccination_v5.pdf. Accessed March 10, 2021.

[41] M Sallam. COVID-19 vaccine hesitancy worldwide: a concise systematic review of vaccine acceptance rates. Vaccines, 9(2), 2021. doi: 10.3390/vaccines9020160. URL https://www. mdpi.com/2076-393X/9/2/160.

[42] The National Institute for Public Health and the Environment (RIVM). Research on behavioural rules and well-being: round 15, 2021. URL https://www.rivm.nl/en/coronavirus-covid-19/research/behaviour/ behavioural-rules-and-well-being-round-15. Accessed January 27, 2022.

[43] The National Institute for Public Health and the Environment (RIVM). Research on behavioural rules and well-being: round 15, $2021 . \quad$ URL https://www.rivm.nl/en/coronavirus-covid-19/research/behaviour/ behavioural-rules-and-well-being-round-15. Accessed January 27, 2022.

[44] IM Rosenstock. The health belief model and preventive health behavior. Health Education Monographs, 2(4):354-386, 1974. doi: 10.1177/109019817400200405. URL https://doi .org/ 10.1177/109019817400200405.

[45] The national institute for public health and the environment (rivm). explanations for (non-)compliance with covid-19 prevention measures, 2021. URL https://www.rivm.nl/ gedragsonderzoek/maatregelen-welbevinden/verklaringen-gedrag. Accessed January 21, 2021.

[46] N Perra, D Balcan, B Gonçalves, and A Vespignani. Towards a characterization of behaviordisease models. PLoS ONE, 6(8):1-15, 2011. doi: 10.1371/journal.pone.0023084. URL https: //journals.plos.org/plosone/article?id=10.1371/journal. pone. 0023084.

[47] SA Pedro, FT Ndjomatchoua, P Jentsch, JM Tchuenche, M Anand, and CT Bauch. Conditions for a Second Wave of COVID-19 Due to Interactions Between Disease Dynamics and Social Processes. Frontiers in Physics, 8:428, 2020. doi: 10.3389/fphy.2020.574514. URL https://www.frontiersin.org/article/10.3389/fphy.2020.574514.

[48] A Teslya, TM Pham, NG Godijk, ME Kretzschmar, MCJ Bootsma, and G Rozhnova. Impact of self-imposed prevention measures and short-term government-imposed social distancing on mitigating and delaying a COVID-19 epidemic: A modelling study. PLOS Medicine, 17(7): 1-21, 2020. doi: 10.1371/journal.pmed.1003166. URL https://doi.org/10.1371/journal. pmed.1003166. 
[49] Rapid risk assessment: Assessing SARS-CoV-2 circulation, variants of concern, non-pharmaceutical interventions and vaccine rollout in the EU/EEA, 15th update, 2021. URL https://www.ecdc.europa.eu/sites/default/files/documents/ RRA-15th-update-June\%202021.pdf. Accessed August 1, 2021.

[50] No choice but to take summertime measures in face of rapid increase in infections: News item: Government.nl, 2021. URL https://www. government.nl/latest/news/2021/07/09/ no-choice-but-to-take-summertime-measures-in-face-of-rapid-increase-in-infections. Accessed September 8, 2021.

[51] ERA Vos, G den Hartog, RM Schepp, P Kaaijk, J van Vliet, K Helm, et al. Nationwide seroprevalence of SARS-CoV-2 and identification of risk factors in the general population of the Netherlands during the first epidemic wave. Journal of Epidemiology 83 Community Health, 2020. doi: 10.1136/jech-2020-215678. URL https://jech.bmj . com/content/early/ 2020/11/28/jech-2020-215678.

[52] The National Institute for Public Health and the Environment (RIVM). PIENTER Corona Studie: Resultaten, 2021. URL https://www.rivm.nl/pienter-corona-studie/ resultaten. Accessed August 1, 2021.

[53] The National Institute for Public Health and the Environment (RIVM). Research on behavioural rules and well-being: round 8, 2021. URL https://www.rivm.nl/en/novel-coronavirus-covid-19/research/behaviour/ -behavioural-rules-and-well-being-round-8. Accessed February 25, 2021.

[54] The National Institute for Public Health and the Environment (RIVM). Epidemiologische situatie COVID-19 in Nederland (17 november 2020), 2020. URL https://www.rivm.nl/sites/ default/files/2020-11/COVID-19_WebSite_rapport_wekelijks_20201117_1237.pdf.

Accessed March 4, 2021.

[55] F Campbell, B Archer, H Laurenson-Schafer, Y Jinnai, F Konings, N Batra, et al. Increased transmissibility and global spread of SARS-CoV-2 variants of concern as at June 2021. Eurosurveillance, 26(24), 2021. doi: https://doi.org/10.2807/1560-7917.ES.2021.26. 24.2100509. URL https://www .eurosurveillance.org/content/10.2807/1560-7917. ES . 2021.26 .24 .2100509 .

[56] JA Backer, L Mollema, ERA Vos, D Klinkenberg, FRM van der Klis, HE de Melker, et al. Impact of physical distancing measures against COVID-19 on contacts and mixing patterns: repeated cross-sectional surveys, the Netherlands, 2016-17, April 2020 and June 2020. Eurosurveillance, 26(8):2000994, 2021. doi: 10.2807/1560-7917.ES.2021.26.8.2000994. URL https: //www. eurosurveillance.org/content/10.2807/1560-7917.ES.2021.26.8.2000994.

[57] S Cuschieri, A Hatziyianni, M Kantaris, A Kontemeniotis, M Theodorou, and E Pallari. Same pandemic yet different COVID-19 vaccination roll-Out rates in two small European islands: a comparison between Cyprus and Malta. Healthcare, 10(2), 2022. ISSN 2227-9032. doi: 10.3390/healthcare10020222. URL https://www.mdpi.com/2227-9032/10/2/222.

[58] N Gozzi, P Bajardi, and N Perra. The importance of non-pharmaceutical interventions during the COVID-19 vaccine rollout. PLOS Computational Biology, 17(9):1-24, 09 2021. doi: 10.1371/journal.pcbi.1009346. URL https://doi.org/10.1371/journal.pcbi.1009346. 
[59] N Gozzi, P Bajardi, and N Perra. The importance of non-pharmaceutical interventions during the COVID-19 vaccine rollout. medRxiv, 2021. doi: 10.1101/2021.01.09.21249480. URL https://www.medrxiv.org/content/early/2021/01/09/2021.01.09.21249480.

[60] S Moore, EM Hill, MJ Tildesley, L Dyson, and MJ Keeling. Vaccination and nonpharmaceutical interventions for COVID-19: a mathematical modelling study. The Lancet Infectious Diseases, 2021. doi: 10.1016/S1473-3099(21)00143-2. URL https://www. thelancet.com/journals/laninf/article/PIIS1473-3099(21)00143-2/fulltext.

[61] S Contreras and V Priesemann. Risking further COVID-19 waves despite vaccination. The Lancet Infectious Diseases, 2021. doi: 10.1016/S1473-3099(21)00167-5. URL https://www . thelancet.com/journals/laninf/article/PIIS1473-30992100167-5/fulltext?

[62] R Rajaraman, Z Sun, R Sundaram, and AKS Vullikanti. Network effects of risk behavior change following prophylactic interventions. PLoS One, 8, 2013. doi: 10.1371/ journal.pone.0064653. URL https://journals.plos.org/plosone/article?id=10.1371/ journal.pone. 0064653.

[63] TN Vilches, M Jaberi-Douraki, and SM Moghadas. Risk of influenza infection with low vaccine effectiveness: the role of avoidance behaviour. Epidemiology and Infection, 147, 2019. doi: 10.1017/S0950268818003540. URL https://pubmed.ncbi.nlm.nih.gov/30869007/.

[64] N Dagan, N Barda, E Kepten, O Miron, S Perchik, MA Katz, et al. BNT162b2 mRNA Covid19 vaccine in a nationwide mass vaccination setting. New England Journal of Medicine, 2021. doi: 10.1056/NEJMoa2101765. URL https://doi.org/10.1056/NEJMoa2101765.

[65] MG Thompson, JL Burgess, AL Naleway, HL Tyner, SK Yoon, J Meece, et al. Interim estimates of vaccine effectiveness of BNT162b2 and mRNA-1273 COVID-19 vaccines in preventing SARS-CoV-2 infection among health care personnel, first responders, and other essential and frontline workers - eight U.S. locations, December 2020-March 2021. Centers for Disease Control and Prevention. Morbidity and Mortality Weekly Report, pages 495-500, 2021. doi: 10.15585/mmwr.mm7013e3. URL https ://www. cdc.gov/mmwr/volumes/70/wr/mm7013e3. htm. Accessed March 31, 2021.

[66] J Lopez Bernal, N Andrews, C Gower, E Gallagher, R Simmons, S Thelwall, et al. Effectiveness of Covid-19 Vaccines against the B.1.617.2 (Delta) Variant. New England Journal of Medicine, 385(7):585-594, 2021. doi: 10.1056/NEJMoa2108891. URL https ://doi .org/10. 1056/NEJMoa2108891.

[67] MD Swift, LE Breeher, AJ Tande, CP Tommaso, CM Hainy, H Chu, et al. Effectiveness of Messenger RNA Coronavirus Disease 2019 (COVID-19) Vaccines Against Severe Acute Respiratory Syndrome Coronavirus 2 (SARS-CoV-2) Infection in a Cohort of Healthcare Personnel. Clinical Infectious Diseases, 04 2021. ISSN 1058-4838. doi: 10.1093/cid/ciab361. URL https://doi.org/10.1093/cid/ciab361. ciab361.

[68] Q Li, X Guan, P Wu, X Wang, L Zhou, Y Tong, et al. Early transmission dynamics in Wuhan, China, of novel coronavirus-infected pneumonia. New England Journal of Medicine, 382(13): 1199-1207, 2020. doi: 10.1056/NEJMoa2001316. URL https://www.nejm.org/doi/full/ 10.1056/NEJMoa2001316. 
[69] M Park, AR Cook, JT Lim, Y Sun, and BL Dickens. A systematic review of COVID-19 epidemiology based on current evidence. Journal of Clinical Medicine, 9(4):967, 2020. doi: 10.3390/jcm9040967. URL https://www.mdpi.com/2077-0383/9/4/967.

[70] O Diekmann, H Heesterbeek, and H Britton. Mathematical Tools for Understanding Infectious Disease Dynamics. Princeton University Press, 2013. URL http://www. jstor.org/stable/ j.cttq9530.

[71] JA Backer, D Klinkenberg, and J Wallinga. Incubation period of 2019 novel coronavirus (2019-nCoV) infections among travellers from Wuhan, China, 20-28 January 2020. Eurosurveillance, 25(5), 2020. doi: 10.2807/1560-7917.ES.2020.25.5.2000062. URL https: //www . eurosurveillance.org/content/10.2807/1560-7917.ES.2020.25.5.2000062.

[72] G Rozhnova, CH van Dorp, P Bruijning-Verhagen, MCJ Bootsma, JHHM van de Wijgert, MJM Bonten, and ME Kretzschmar. Model-based evaluation of school- and non-school-related measures to control the COVID-19 pandemic. Nature Communications, 12, 2021.

[73] MATLAB. 9.9.0.1570001 (R2020b) Update 4. The MathWorks Inc., Natick, Massachusetts, 2020.

[74] A Teslya, G Rozhnova, TM Pham, D van Wees, H Nunner, NG Godijk, and ME Bootsma, MCJ Kretzschmar. The importance of sustained compliance with physical distancing during COVID-19 vaccination rollout. GitHub, 2022. doi: 10.5281/zenodo.5925103. URL https: //github.com/aiteslya/VaccineCompliance1.2. 


\section{Supplementary Files}

This is a list of supplementary files associated with this preprint. Click to download.

- SupplementaryMaterial.pdf 